\title{
Ethnopharmacological survey among migrants living in the Southeast Atlantic Forest of Diadema, São Paulo, Brazil
}

\author{
Daniel Garcia"* ${ }^{*}$ Marcus Vinicius Domingues ${ }^{1}$, Eliana Rodrigues ${ }^{2}$
}

\begin{abstract}
Background: Understanding how people of diverse cultural backgrounds have traditionally used plants and animals as medicinal substances during displacements is one of the most important objectives of ethnopharmacological studies. An ethnopharmacological survey conducted among migrants living in the Southeast Atlantic Forest remnants (Diadema, São Paulo, Brazil) is presented herein.

Methods: Ethnographical methods were used to select and interview the migrants, and botanical and zoological techniques were employed to collect the indicated resources.

Results: We interviewed five migrants who described knowledge on 12 animals and 85 plants. Only 78 plants were present in Diadema, they belong to 37 taxonomic families; 68 were used exclusively for medicinal purposes, whereas 10 were reported to be toxic and/or presented some restriction of use. These taxa were grouped into 12 therapeutic categories (e.g., gastrointestinal disturbances, inflammatory processes or respiratory problems) based on the 41 individual complaints cited by the migrants. While the twelve animal species were used by the migrants to treat nine complaints; these were divided into six categories, the largest of which related to respiratory problems. None of the animal species and only 57 of the 78 plant species analysed in the present study were previously reported in the pharmacological literature; the popular knowledge concurred with academic findings for 30 of the plants. The seven plants [Impatiens hawkeri W. Bull., Artemisia canphorata Vill., Equisetum arvensis L., Senna pendula (Humb. \& Bonpl. ex Willd.) H.S. Irwin \& Barneby, Zea mays L., Fevillea passiflora Vell. and Croton fuscescens Spreng)] and the two animals (Atta sexdens and Periplaneta americana) that showed maintenance of use among migrants during their displacement in Brazilian territory, have not been studied by pharmacologists yet.
\end{abstract}

Conclusions: Thus, they should be highlighted and focused in further pharmacology and phytochemical studies, since the persistence of their uses can be indicative of bioactive potentials.

\section{Background}

Cultural mixing mediated by the migration of people around the world has generated increasing interest in recent years within the field of ethnopharmacology [1]. Medicinal plants have been used by human societies throughout history, also across geographical barriers [2]. The continuous use of certain plants and animals for medicinal purposes over time reflects their potential therapeutic value. Such substances become even more promising when they are persistently used by migrating

\footnotetext{
* Correspondence: danielgarciafic@hotmail.com

'Department of Biology, Universidade Federal de São Paulo, Rua Arthur Ridel, 275 CEP, 09941-510, Diadema, S.P., Brazil

Full list of author information is available at the end of the article
}

human groups despite the considerable distances travelled and the consequent exposure to different cultures and vegetal resources. Numerous studies have collected information on medicinal plants from ethnic groups who migrated from Mexico to the U.S.A. [3,4]; from Haiti to Cuba [5]; from Africa to South America [6]; from Africa to Brazil [7]; from Colombia to London [8]; from Suriname to the Netherlands [9]; from Albania to southern Italy [10,11]; from Germany to eastern Italy [12]; and from Europe and Africa to eastern Cuba $[1,13]$. However, few studies have focused on migration within a country, such as that described by Rodrigues et al. [14] regarding migrants from northeastern Brazil who currently occupy the southeast.

\section{C) Biomed Central}


Brazil offers a favourable environment for studies focused on migration and medicinal plants/animals because it possesses a large area of $8,514,876.599 \mathrm{~km}^{2}$ [15] and boasts high indices of cultural and biological diversity. Brazil is inhabited by rural and urban populations of 232 indigenous ethnic groups [16], 1,342 Quilombola groups (descendants of Afro-Brazilian people) [17], and mestizo groups derived from the miscegenation of Indian, Black, European and Asiatic people. Brazil also houses 55,000 species of higher plants [18] and almost $7 \%$ of global animal diversity was described (ca. 100,000 out of 1.5 million), though some estimates suggest that this number is significantly higher [19]. Migration between regions of this country encourages contact with the rich biological and cultural diversity and allows interpersonal interactions that contribute to the transformation of local medicinal therapies.

According to Simões and Lino [20], the original Atlantic Forest covered approximately 1.3 million $\mathrm{km}^{2}$, spanning 17 Brazilian states from south to northeast; however, it currently covers only 14 states, and its area has been reduced to $65,000 \mathrm{~km}^{2}$. Despite considerable fragmentation, the Atlantic Forest still contains more than 20,000 plant species (8,000 endemic) and 1,361 animal species (567 endemic). It is the richest forest in the world in wood plants per unit area; the southern Bahia, for example, holds a record of 454 different species/ha [21].

The objective of this study was to perform an ethnopharmacological survey among migrants from northeastern and southeastern Brazil who currently live in Atlantic Forest remnants in the municipality of Diadema (São Paulo state, southeastern Brazil). We attempted to understand how the medicinal use of certain plant and animal changed as a result of the migrants' contact with new therapies, diseases and natural resources found in Diadema. These findings were classified as either: maintenance, replacement, incorporation or discontinuation of plants/animals use.

These objectives are in agreement with several stated goals of ethnopharmacology, namely, to investigate how migration can influence knowledge of medicinal plants/ animals, the extent to which displaced people incorporate new species into their therapeutic methods, and, in particular, why individuals sometimes persistently adhere to old customs, before or even after they are exposed to new possibilities. Therefore, we adopt the hypothesis that the use of plants/animals as medicines is influenced by migratory movements, and access to natural resources available in the municipality of Diadema.

\section{Methodology}

\section{Fieldwork}

One of the authors (D. Garcia) spent 14 months (September 2007 to November 2008) in the municipality of

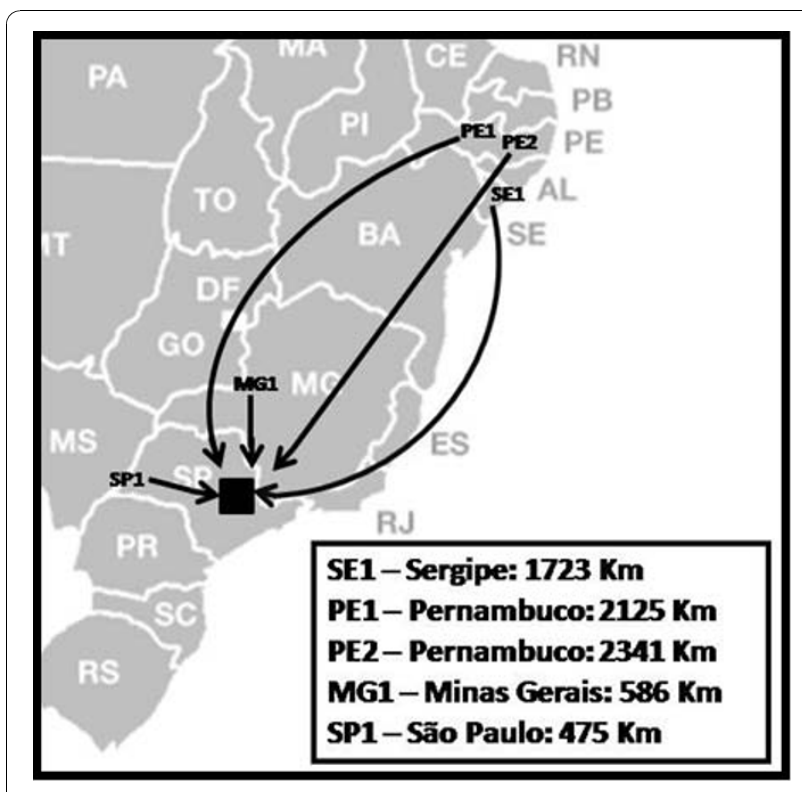

Figure 1 Location of the Municipality of Diadema, in São Paulo state, southeastern Brazil (black square). Interviewees' migration from their cities of origin to Diadema, being PE (Pernambuco state), SE (Sergipe), MG (Minas Gerais) and SP (São Paulo), and the distance of the displacement in each case (in $\mathrm{Km}$ ).

Diadema, São Paulo, SP, Brazil (2341'10"S, 46³7’22"W) (Figure 1), selecting, observing and interviewing migrants living in the Atlantic Forest remnants. Diadema is located 16 kilometres from the capital São Paulo, covers an area of $30.65 \mathrm{~km}^{2}$, and is occupied by 394.266 inhabitants [15], most of whom are migrants from other regions of Brazil. The municipality has a literacy rate of $6.8 \%$ [22], and its Human Development Index is 0.79 [23]. The Atlantic Forest remnants found in this city are rich in plants that are either native or introduced by the influence of those migrants present both in urban and rural areas.

Migrants who had relevant knowledge regarding the use of plants and animals for medicinal purposes were selected for interviews following the purposive sampling method [24]. Thus, we sought information about the presence of migrants from herb traders, health care professionals, and some local prayer-makers. According to Bernard [24], this sampling is also known as judgment sampling, utilized during qualitative research in particular in those populations that are difficult to be localized, the researcher selects interviewees based on their trial that they meet the criteria for the study of the phenomenon of interest. After identifying potential interviewees, the researcher visited them to determine whether they did indeed possess knowledge on medicinal plants and whether they wanted to take part in this study. The ethnopharmacological study was approved by the Ethics Committee of Universidade Federal de São Paulo 
(UNIFESP's Ethics Committee on Research 1969/07) and Conselho de Gestão do Patrimônio Genético (No. 02000.001 049/2008-71). The interviewees also signed consent forms granting permission to access their knowledge and collect botanical and zoological material.

Personal and ethnopharmacological data from the interviewees were obtained through informal and semistructured interviews [24] that addressed the following topics: personal details and migration history (name, sex, age, religion, marital status, place of birth, migration, main occupation, grade of schooling) as well as ethnopharmacology (name of natural resource, use, part used, formula, route of administration, contraindications, dosages, restrictions of use). The author (D. Garcia) has visited each interviewee at least 12 times, in order to fill in the forms mentioned above, as well as to understand their processes of acquiring knowledge in depth.

Each medicinal plant was collected in the presence of the person who described it during the interviews, in accordance with the methods suggested by Lipp [25]. The plants' scientific names were determined by specialists from the Instituto de Botânica do Estado de São Paulo (IB), and vouchers were deposited at the Herbário Municipal de São Paulo (PMSP). The animals collected were placed in glass vials containing $70 \%$ ethyl alcohol, and their subsequent identification and deposit were performed by zoologists from the Museum of Zoology, Universidade de São Paulo (MZUSP) and the Bioscience Institute from Universidade de São Paulo (IB-USP).

When interviewees cited plants and animals that were used only in their cities of origin, i.e., not available in Diadema, photos from the literature and other information (e.g., popular name, habits and habitat) were used to identify them to at least the genus level. These organisms are marked with asterisks throughout the text and in Table 1. The Herpetofauna of the Northeast Atlantic Forest [26] and The Herpetofauna of Caatingas and Altitudes Areas of the Brazilian Northeast [27] were used as identification guides. For plants, we also consulted Medicinal Plants in Brazil - Native and Exotic [28].

\section{Database survey}

For the plants and animals identified to the species level, we searched the bibliographic databases PUBMED [29] and SCIFINDER [30] to determine whether they had been targets of previous pharmacological studies. To determine the origin of each plant species, we consulted the Dictionary of Useful Plants: exotic and native [31].

\section{Dynamics of use}

During our field work, we made an effort to understand the dynamics of use for each resource and classified them into the following four categories: maintenance of use (resource used for the same purpose in the migrant's city of origin and in Diadema), replacement (resources that were replaced when migrants arrived in Diadema because the original product was not available in Diadema or was less effective than the new resource), incorporation (resources used for the first time in Diadema to treat diseases common to larger cities, such as hypertension, diabetes and anxiety, which were not common in their homeland), and finally discontinued use (resources that are no longer used in Diadema, usually because they are not available).

\section{Data analysis}

The level of homogeneity between plant information provided by different migrants was calculated using the Informants' Consensus Factor, Fic [32]. This term is calculated as Fic $=N u r-N t /(N u r-1)$, where Nur is the number of use reports from informants for a particular plant-usage category and $N t$ is the number of taxa or species used for that plant usage category across all informants. Values range between 0 and 1 , with 1 indicating the highest level of informant consent. For instance, if certain taxa are consistently used by informants, then a high degree of consensus is reached and medicinal traditions are viewed as well-defined [33].

\section{Results and Discussion}

\section{Migrant Interviews}

Despite the fact that Diadema is composed by thousands of migrants, we observed that only a few have retained traditional knowledge pertaining to medicinal plants and animals. Some considerations should be made, in order to justify our decision of conducing a qualitative approach, in depth, with the sample of interviewees obtained during the two months prior to the start of the study. During this time we observed that in many cases, this knowledge has fallen into disuse because of: a) a cultural adaptation to the new city, b) the ease of conventional medical care, c) forest degradation, which restricts use of local plants and animals, furthermore d) many migrants have shown concern to participate in the study, since in the past they suffered persecution from government agencies and physicians, who eventually restrained their medical practice.

The five selected interviewees migrated from northeast and southeast Brazil and established themselves in Diadema in the $1940 \mathrm{~s}$. Three were born in the northeast: two in Pernambuco state (coded as PE1 and PE2) and one in Sergipe state (SE1). The two remaining migrants were born in the southeast: one in Minas Gerais state (MG1) and one in inland São Paulo state (SP1) (Figure 1). All interviewees were Catholic, married and retired, with the exception of PE1 and PE2 who sell medicinal plants. Their average age was approximately 68 years old (ranging from 53 to 80 years old), and their level of 
Table 1 The 12 animals indicated by migrant PE2, their popular and scientific names, complaints (part used), formula and route of administration

\begin{tabular}{|c|c|c|}
\hline Popular name dynamic of use & $\begin{array}{l}\text { Scientific name or only genus (family/class) } \\
\text { Voucher }\end{array}$ & Complaint (part used) - formula - route of administration \\
\hline 1- Snake (cobra) ${ }^{\circ}$ & $\begin{array}{l}\text { Chironius sp., Liophs sp. (Colubridae/Reptilia)* or } \\
\text { Bothops sp. (Viperidae/Reptilia)* }\end{array}$ & Bronchitis (skin) - powder - ingested \\
\hline \multirow[t]{3}{*}{ 2- Rattlesnake (cascavel) ${ }^{\circ}$} & Crotalus cf. durissus L. (Viperidae/Reptilia)* & Back pain (fat) - in natura - ingested \\
\hline & & Bronchitis (rattle) - tie it in the neck - topic \\
\hline & & Heart problems (tooth) - put it in the pocket of shirt \\
\hline $\begin{array}{l}\text { 3- Cururu frog (sapo- } \\
\text { cururu) }^{\circ}\end{array}$ & Rhinella sp. (Bufonidae/Amphibia)* & $\begin{array}{l}\text { Cancer of skin (whole animal) - in natura: tie it on the cancer } \\
\text { for some time each day - topic }\end{array}$ \\
\hline \multirow[t]{2}{*}{ 4- Alligator (jacaré) ${ }^{\circ}$} & $\begin{array}{l}\text { Crocodilus sp., Cayman sp. or Paleosuchus sp. } \\
\text { (Alligatoridae/Reptilia)* }\end{array}$ & Apoplexy (skin) - syrup of skin powder - ingested \\
\hline & & Bronchitis (bone) - powder - ingested \\
\hline 5- Turtle (tartaruga) ${ }^{\circ}$ & Geochelone sp. (Testudinidae/Reptilia)* & Bronchitis and asthma - (turtleshell) - powder - ingested \\
\hline 6- Capybara (capivara) ${ }^{\circ}$ & $\begin{array}{l}\text { Hydrochoerus cf. hydrochaeris L. (Hydrochaeridae/ } \\
\text { Mammalia)* }\end{array}$ & Bronchitis and asthma - (skin) - powder - ingested \\
\hline 7 -Iguana (iguana) ${ }^{\circ}$ & Iguana cf. iguana L. (Iguanidae/Reptilia) * & Osteoporosis and rheumatism (bone) - powder - ingested \\
\hline 8- Ant (formiga) ${ }^{\square}$ & Atta sexdens L. (Formicidae/Insecta) Garcia 001 & Epilepsy (anthill) - in natura - ingested \\
\hline 9- Cockroach (barata) & $\begin{array}{l}\text { Periplaneta americana L. (Blattidae/Insecta) } \\
\text { Garcia } 002\end{array}$ & Bronchitis and asthma (whole animal) - powder - ingested \\
\hline $\begin{array}{l}\text { 10- Water cockroach } \\
\text { (barata d'água) }^{\circ}\end{array}$ & $\begin{array}{l}\text { Abedus sp., Belostoma sp. or Diplonychus sp. } \\
\text { (Belostomatidae/Insecta)* }\end{array}$ & Bronchitis and asthma (whole animal) - powder - ingested \\
\hline 11- Lizard (calango) ${ }^{\circ}$ & Placosoma sp. (Gymnophthalmidae/Reptilia)* & Wounds in the body (skin) - powder - ingested \\
\hline $\begin{array}{l}\text { 12- Armadillo-ball (tatu- } \\
\text { bola) }\end{array}$ & Tolypeutes sp. (Dasypodidae/Mammalia)* & Wounds in the body (skin) - powder - ingested \\
\hline
\end{tabular}

Marked by $\left({ }^{\square}\right)$ the two animals whose use had been maintained, while 10 , marked by $\left(^{\circ}\right)$ are those whose uses have fallen into disuse.

* Animals that couldn't be collected because were not available in Diadema.

education was semi-illiterate to illiterate. They learned about the medicinal uses of plants and animals from their parents and grandparents (Brazilian natives, European and African descendants) in their homelands. All interviewees arrived in the city of Diadema as adults, and some had migrated through different regions of Brazil, accumulating knowledge on natural resources from human and biological sources. In Diadema, they acquired knowledge from neighbours, books, media (radio, television, magazines), and personal experiences.

\section{Plants: dynamics of use}

The migrants described their knowledge of 85 plant specimens. As can be seen in Table 2, 78 of them were available in Diadema and were collected, resulting in 65 plant species, the remaining 13 could only be identified to the generic level. The plants belong to 37 taxonomic families, with Asteraceae (16 species), Lamiaceae (8) and Euphorbiaceae (7) as the most common. Previous studies have shown that Asteraceae species are the group most commonly reported to have potential pharmacological properties, not only in the Atlantic Forest [34-36] but also in other Brazilian biomes such as the Amazon Forest [37] the pantanal wetlands [38] and the cerrado savannahs [39]. In a review focusing on plants with possible action/ effects on the central nervous system that were indicated by 26 Brazilian indigenous peoples occupying different Brazilian biomes [14], Asteraceae was the second most commonly cited family. The same pattern has been detected in other countries, such as Mexico [40]. One factor that may explain the common use of this taxonomic family is the large number of species belonging to it - about 20,000 [41]. Asteraceae also has a wide geographical distribution, both in Brazil and throughout the world [42], which facilitates its use by various cultures.

From the 65 species identified, it was observed that 33 are native to Brazil while the other 32 are exotic, demonstrating the great floral diversity of the region, which was influenced by European and African people during the civilizing process in Brazil. Furthermore, of the 78 specimens recorded, 54\% (42) are spontaneous or were already available in Diadema when interviewees arrived there, while $46 \%$ (36) were grown by the migrants, acquired in free markets, or brought from other regions of the country during migration. Below, we describe the four 'dynamics of use' categories observed during this study.

\section{Maintenance of use}

According to the interviewees, 68 of the 78 specimens cited in the present study, were used in their homelands 
Table 2 The 78 plant specimens used by five Diadema's migrants (MG1, SP1, PE1, PE2, SE1)*.

\begin{tabular}{|c|c|c|c|c|c|}
\hline $\begin{array}{l}\text { Popular(s) } \\
\text { name(s) } \\
\text { (migrant) dynamic } \\
\text { of use }\end{array}$ & $\begin{array}{l}\text { Specimen (family) } \\
\text { Voucher }\end{array}$ & $\begin{array}{l}\text { Origin - } \\
\text { geographical } \\
\text { distribution - } \\
\text { cultivated (C) or } \\
\text { spontaneous (S) }\end{array}$ & Use (part) & $\begin{array}{l}\text { Formula and } \\
\text { route of } \\
\text { administration }\end{array}$ & Pharmacological studies \\
\hline $\begin{array}{l}\text { 1-Alamanda- } \\
\text { amarela } \\
\left(\mathrm{SE} 1^{\square}, \mathrm{PE}^{\triangle}\right) \\
\end{array}$ & $\begin{array}{l}\text { Allamanda cathartica } \\
\text { L. (Apocynaceae) } \\
\text { Garcia } 076\end{array}$ & $\begin{array}{l}\text { Native - Brazilian } \\
\text { territory (C) }\end{array}$ & $\begin{array}{l}\text { Toxic (whole } \\
\text { plant) }\end{array}$ & $\begin{array}{l}\text { Any oral dose is } \\
\text { dangerous }\end{array}$ & Healing activity [65] \\
\hline 2-Alecrim (MG1) & $\begin{array}{l}\text { Rosmarinus officinalis } \\
\text { L. (Lamiaceae) } \\
\text { Garcia } 060\end{array}$ & $\begin{array}{l}\text { Exotic - all countries } \\
\text { with temperate } \\
\text { climate }(C)\end{array}$ & $\begin{array}{l}\text { Muscle pain* } \\
\text { (leaves) }\end{array}$ & $\begin{array}{l}\text { Decoction - } \\
\text { massage }\end{array}$ & $\begin{array}{l}\text { Antibacterial effects [66], antimicrobial } \\
\text { effect [67], anti-inflammatory and anti- } \\
\text { tumor effects [68], cause reduction of } \\
\text { reproductive fertility in male rats [69], } \\
\text { antinociceptive effect [70], mosquito } \\
\text { repellent activity [71], antidiabetic and } \\
\text { antioxidant properties [72] }\end{array}$ \\
\hline $\begin{array}{l}\text { 3-Alecrim-do- } \\
\text { campo } \\
(\mathrm{SE} 1)^{\square}\end{array}$ & $\begin{array}{l}\text { Baccharis } \\
\text { dracunculifolia DC } \\
\text { (Asteraceae) } \\
\text { Garcia } 021\end{array}$ & $\begin{array}{l}\text { Native - central Brazil } \\
\text { (S) }\end{array}$ & $\begin{array}{l}\text { Soothing (aerial } \\
\text { parts) }\end{array}$ & $\begin{array}{l}\text { Smoking - } \\
\text { inhalation }\end{array}$ & $\begin{array}{l}\text { Bactericidal activity }[73] \text {, cytotoxic }[74], \\
\text { antiulcerogenic }[75] \text {, antimicrobial and } \\
\text { antifungal }[76] \text { and anti-inflammatory } \\
{[77]}\end{array}$ \\
\hline \multirow[t]{2}{*}{$\begin{array}{l}\text { 4-Alfavaca } \\
(\mathrm{SP} 1)^{\square}\end{array}$} & $\begin{array}{l}\text { Ocimum selloi Benth. } \\
\text { (Lamiaceae) } \\
\text { Garcia } 033\end{array}$ & $\begin{array}{l}\text { Native - northeast to } \\
\text { south Brazil (C) }\end{array}$ & $\begin{array}{l}\text { Soothing (aerial } \\
\text { parts) }\end{array}$ & $\begin{array}{l}\text { Infusion - } \\
\text { inhalation }\end{array}$ & Mosquito repellent activity [78] \\
\hline & & & Bronchitis (leaves) & Syrup - ingestion & \\
\hline $\begin{array}{l}\text { 5-Algodão } \\
(M G 1)^{\square}\end{array}$ & $\begin{array}{l}\text { Gossypium sp. } \\
\text { (Malvaceae) } \\
\text { Garcia } 066 \\
\end{array}$ & No data $(\mathrm{C})$ & $\begin{array}{l}\text { Anti-inflammatory } \\
\text { (leaves) }\end{array}$ & $\begin{array}{l}\text { Infusion - } \\
\text { inhalation }\end{array}$ & Not consulted \\
\hline $\begin{array}{l}\text { 6-Algodão-do- } \\
\text { mato } \\
(\mathrm{MG} 1, \mathrm{PE} 2)^{\square}\end{array}$ & $\begin{array}{l}\text { Asclepias curassavica } \\
\text { L. (Apocynaceae) } \\
\text { Garcia } 037\end{array}$ & $\begin{array}{l}\text { Exotic - Brazilian } \\
\text { territory }(\mathrm{S})\end{array}$ & $\begin{array}{l}\text { Toxic* (whole } \\
\text { plant) }\end{array}$ & $\begin{array}{l}\text { Any oral dose is } \\
\text { dangerous }\end{array}$ & $\begin{array}{l}\text { Cancer and warts treatment }[79] \text { and } \\
\text { poisoning }[80]\end{array}$ \\
\hline $\begin{array}{l}\text { 7-Almeirão-boca- } \\
\text { de-leão }(\mathrm{SE} 1)^{\Delta}\end{array}$ & $\begin{array}{l}\text { Hypochoeris sp. } \\
\text { (Asteraceae) } \\
\text { Garcia } 009\end{array}$ & No data (S) & Liver pain (leaves) & $\begin{array}{l}\text { In natura - } \\
\text { ingestion }\end{array}$ & Not consulted \\
\hline $\begin{array}{l}\text { 8-Amendoim- } \\
\text { bravo, burra- } \\
\text { leiteira (MG1, } \\
\text { SP1, SE1, PE1, } \\
\text { PE2) }\end{array}$ & $\begin{array}{l}\text { Euphorbia heterophylla } \\
\text { L. (Euphorbiaceae) } \\
\text { Garcia } 047\end{array}$ & Native - Americas (S) & $\begin{array}{l}\text { Toxic* (whole } \\
\text { plant) }\end{array}$ & $\begin{array}{l}\text { Any oral dose is } \\
\text { dangerous }\end{array}$ & Cytotoxic properties [81] \\
\hline 9-Anador (SE1) & $\begin{array}{l}\text { Alternanthera sp. } \\
\text { (Amaranthaceae) } \\
\text { Garcia } 039\end{array}$ & No data (C) & $\begin{array}{l}\text { Soothing, } \\
\text { headache, pain in } \\
\text { the body (leaves) }\end{array}$ & $\begin{array}{l}\text { Infusion - } \\
\text { ingestion }\end{array}$ & Not consulted \\
\hline$\overline{10-A r n i c a ~(P E 1) ~}$ & $\begin{array}{l}\text { Porophyllum ruderale } \\
\text { (Jacq.) Cass. } \\
\text { (Asteraceae) } \\
\text { Garcia } 075\end{array}$ & $\begin{array}{l}\text { Native - Brazilian } \\
\text { territory }(S)\end{array}$ & $\begin{array}{l}\text { Muscle pain* } \\
\text { (aerial parts) }\end{array}$ & $\begin{array}{l}\text { Decoction - } \\
\text { massage }\end{array}$ & Anti-inflammatory [82] \\
\hline $\begin{array}{l}\text { 11-Aroeira } \\
(\mathrm{MG} 1)^{\square}\end{array}$ & $\begin{array}{l}\text { Schinus terebinthifolius } \\
\text { Raddi (Anacardiaceae) } \\
\text { Garcia } 035\end{array}$ & $\begin{array}{l}\text { Native - northeast to } \\
\text { south Brazil (S) }\end{array}$ & Diuretic (leaves) & $\begin{array}{l}\text { Infusion - } \\
\text { ingestion }\end{array}$ & $\begin{array}{l}\text { Antifungal activity }[83] \text { and antibacterial } \\
{[84]}\end{array}$ \\
\hline \multirow[t]{2}{*}{$\begin{array}{l}\text { 12-Arruda (MG1, } \\
\text { PE1, PE2) }\end{array}$} & $\begin{array}{l}\text { Ruta graveolens L. } \\
\text { (Rutaceae) } \\
\text { Garcia } 028\end{array}$ & $\begin{array}{l}\text { Exotic - Brazilian } \\
\text { territory (C) }\end{array}$ & $\begin{array}{l}\text { Earache and } \\
\text { conjunctivitis/styl* } \\
\text { (leaves) }\end{array}$ & In natura - topic & $\begin{array}{l}\text { Antifertility [85], fungicide [86], cytotoxic } \\
{[87] \text {, abortive [88], anti-tumour [89], anti- }} \\
\text { inflammatory [90], antiarrhythmic [91] } \\
\text { and antimicrobial [92] }\end{array}$ \\
\hline & & & $\begin{array}{l}\text { Muscle pain } \\
\text { (leaves) }\end{array}$ & $\begin{array}{l}\text { Decoction - } \\
\text { massage }\end{array}$ & \\
\hline \multirow[t]{3}{*}{$\begin{array}{l}\text { 13- Assa-peixe } \\
(M G 1, S E 1)^{\square}\end{array}$} & $\begin{array}{l}\text { Vernonia sp. } \\
\text { (Asteraceae) } \\
\text { Garcia } 048\end{array}$ & No data (S) & Bronchitis (leaves) & $\begin{array}{l}\text { Infusion - } \\
\text { ingestion }\end{array}$ & Not consulted \\
\hline & & & $\begin{array}{l}\text { Expectorant } \\
\text { (leaves) }\end{array}$ & $\begin{array}{l}\text { Infusion - } \\
\text { inhalation }\end{array}$ & \\
\hline & & & $\begin{array}{l}\text { Healing wounds } \\
\text { (leaves) }\end{array}$ & infusion - plaster & \\
\hline
\end{tabular}


Table 2: The 78 plant specimens used by five Diadema's migrants (MG1, SP1, PE1, PE2, SE1)*. (Continued)

\begin{tabular}{|c|c|c|c|c|c|}
\hline \multirow[t]{2}{*}{$\begin{array}{l}\text { 14-Avelóz (PE1, } \\
\text { PE2) }\end{array}$} & \multirow[t]{2}{*}{$\begin{array}{l}\text { Euphorbia tirucalli L. } \\
\text { (Euphorbiaceae) } \\
\text { Garcia } 046\end{array}$} & \multirow[t]{2}{*}{$\begin{array}{l}\text { Exotic - Brazilian } \\
\text { territory }(C)\end{array}$} & \multirow{2}{*}{$\begin{array}{l}\text { Toxic* (whole } \\
\text { plant) } \\
\text { Breast cancer* } \\
\text { (latex) }\end{array}$} & \multirow{2}{*}{$\begin{array}{l}\text { Restricted use } \\
\text { (reports of } \\
\text { blindness) } \\
\text { Macerate - } \\
\text { ingestion }\end{array}$} & \multirow[t]{2}{*}{$\begin{array}{l}\text { Anti-tumour activity [93], cause eye } \\
\text { injury [94] and effect against arthritis } \\
\text { diseases [95] }\end{array}$} \\
\hline & & & & & \\
\hline$\overline{15-A z a l e ́ i a ~(P E 1)})^{\triangle}$ & $\begin{array}{l}\text { Rhododendron simsii } \\
\text { Planch. (Ericaceae) } \\
\text { Garcia } 043\end{array}$ & $\begin{array}{l}\text { Exotic - Brazilian } \\
\text { territory }(C)\end{array}$ & $\begin{array}{l}\text { Toxic (whole } \\
\text { plant) }\end{array}$ & $\begin{array}{l}\text { Any oral dose is } \\
\text { dangerous }\end{array}$ & Antioxidative [96] \\
\hline \multirow[t]{2}{*}{$\begin{array}{l}\text { 16-Bálsamo } \\
(M G 1, \text { SP1, PE1, } \\
\text { SE1) }\end{array}$} & $\begin{array}{l}\text { Sedum sp. } \\
\text { (Crassulaceae) } \\
\text { Garcia } 038\end{array}$ & No data (C) & Earache (leaves) & In natura - topic & Not consulted \\
\hline & & & $\begin{array}{l}\text { Laxative (aerial } \\
\text { parts) }\end{array}$ & $\begin{array}{l}\text { In natura - } \\
\text { ingestion }\end{array}$ & \\
\hline $\begin{array}{l}\text { 17-Boldo-do- } \\
\text { Chile, figatil } \\
\left(\mathrm{PE} 1^{\square}, \mathrm{SE}^{\triangle}\right)\end{array}$ & $\begin{array}{l}\text { Vernonia condensata } \\
\text { Baker (Asteraceae) } \\
\text { Garcia } 001\end{array}$ & $\begin{array}{l}\text { Exotic - northeast to } \\
\text { southeast Brazil }(C)\end{array}$ & $\begin{array}{l}\text { Liver pain* } \\
\text { (leaves) }\end{array}$ & $\begin{array}{l}\text { Infusion - } \\
\text { ingestion }\end{array}$ & $\begin{array}{l}\text { Anti-ulcerogenic [97] and analgesic and } \\
\text { anti-inflammatory [98] }\end{array}$ \\
\hline $\begin{array}{l}\text { 18-Brinco-de- } \\
\text { princesa }(S E 1)^{\triangle}\end{array}$ & $\begin{array}{l}\text { Alpinia zerumbet } \\
\text { (Pers.) B.L. Burtt \& R.M. } \\
\text { Sm. (Zingiberaceae) } \\
\text { Garcia } 018\end{array}$ & $\begin{array}{l}\text { Exotic - Brazilian } \\
\text { territory }(C)\end{array}$ & Sedative (flowers) & $\begin{array}{l}\text { Infusion - } \\
\text { ingestion }\end{array}$ & $\begin{array}{l}\text { Antihypertensive effects [99], } \\
\text { antinociceptive [100], anti-amoebic } \\
\text { activity [101] and hepatoprotector [102] }\end{array}$ \\
\hline \multirow[t]{2}{*}{ 19-Café (MG1) } & $\begin{array}{l}\text { Coffea arabica L. } \\
\text { (Rubiaceae) } \\
\text { Garcia } 030\end{array}$ & $\begin{array}{l}\text { Exotic - Brazilian } \\
\text { territory }(C)\end{array}$ & $\begin{array}{l}\text { Diabetes (ripe } \\
\text { fruits) }\end{array}$ & Infusion & Antioxidant [103] \\
\hline & & & $\begin{array}{l}\text { Sinusitis (powder } \\
\text { fruit) }\end{array}$ & Infusion & \\
\hline $\begin{array}{l}\text { 20-Cana-do-brejo } \\
(\mathrm{SP} 1, \mathrm{PE} 2)^{\square}\end{array}$ & $\begin{array}{l}\text { Costus spiralis (Jacq.) } \\
\text { Roscoe (Costaceae) } \\
\text { Garcia } 019\end{array}$ & $\begin{array}{l}\text { Native - northeast } \\
\text { and southeast Brazil } \\
\text { (S) }\end{array}$ & $\begin{array}{l}\text { Laxative and } \\
\text { rheumatism } \\
\text { (leaves) }\end{array}$ & $\begin{array}{l}\text { Infusion or } \\
\text { decoction - } \\
\text { ingestion }\end{array}$ & Antiurolithiatic [104] \\
\hline $\begin{array}{l}\text { 21-Cânfora (MG1, } \\
\text { PE1, SE1) }\end{array}$ & $\begin{array}{l}\text { Artemisia canphorata } \\
\text { Vill. (Asteraceae) } \\
\text { Garcia } 045\end{array}$ & $\begin{array}{l}\text { Exotic - Brazilian } \\
\text { territory }(C)\end{array}$ & $\begin{array}{l}\text { Muscle pain } \\
\text { (whole plant) }\end{array}$ & $\begin{array}{l}\text { Decoction - } \\
\text { massage }\end{array}$ & No data found \\
\hline \multirow[t]{2}{*}{$\begin{array}{l}\text { 22-Capim-limão } \\
\text { (MG1, SE1, } \\
\text { PE2) }\end{array}$} & $\begin{array}{l}\text { Cymbopogon citratus } \\
\text { DC. - Stapf. (Poaceae) } \\
\text { Garcia } 026\end{array}$ & $\begin{array}{l}\text { Exotic - tropical } \\
\text { countries }(C)\end{array}$ & $\begin{array}{l}\text { Bronchitis* } \\
\text { (leaves) }\end{array}$ & Syrup - ingestion & $\begin{array}{l}\text { Anxiolytic [105], larvicidal activity [106], } \\
\text { antibacterial [107], antimalarial activity } \\
\text { [108], insect repellent [109], } \\
\text { hypoglycemic and hypolipidemic } \\
\text { effects [110]and antimicrobial activity } \\
\text { [92] }\end{array}$ \\
\hline & & & Sedative* (leaves) & $\begin{array}{l}\text { Infusion - } \\
\text { ingestion }\end{array}$ & \\
\hline $\begin{array}{l}\text { 23-Capuchinha } \\
(\mathrm{SP} 1, \mathrm{MG} 1)^{\triangle}\end{array}$ & $\begin{array}{l}\text { Tropaeolum majus L. } \\
\text { (Tropaeolaceae) } \\
\text { Garcia } 057\end{array}$ & $\begin{array}{l}\text { Exotic - south and } \\
\text { southeast Brazil }(C)\end{array}$ & $\begin{array}{l}\text { Ulcer and laxative } \\
\text { (aerial parts) }\end{array}$ & $\begin{array}{l}\text { Infusion or in } \\
\text { natura - } \\
\text { ingestion }\end{array}$ & Antitumor activity [111] \\
\hline $\begin{array}{l}\text { 24-Carqueja } \\
(\mathrm{MG} 1)^{\square}\end{array}$ & $\begin{array}{l}\text { Baccharis trimera } \\
\text { (Less) DC (Asteraceae) } \\
\text { Garcia } 027\end{array}$ & $\begin{array}{l}\text { Native - south and } \\
\text { southeast Brazil (C) }\end{array}$ & $\begin{array}{l}\text { Diabetes* (whole } \\
\text { plant) }\end{array}$ & $\begin{array}{l}\text { Macerate - } \\
\text { ingestion }\end{array}$ & $\begin{array}{l}\text { Antihepatotoxic properties [112], anti- } \\
\text { inflammatory and analgesic activity } \\
{[113] \text {, relaxant effect }[114] \text {, anti- }} \\
\text { proteolytic and anti-hemorrhagic } \\
\text { properties [115], antioxidant } \\
\text { compounds [116], antidiabetic activity } \\
\text { [117]and for losing weight [118] }\end{array}$ \\
\hline $\begin{array}{l}\text { 25-Carrapicho } \\
\left(\mathrm{SE} 1^{\#}, \mathrm{MG}^{\square}\right)\end{array}$ & $\begin{array}{l}\text { Acanthospermum } \\
\text { australe (Loefl.) Kuntze } \\
\text { (Asteraceae) } \\
\text { Garcia } 052\end{array}$ & $\begin{array}{l}\text { Native - Brazilian } \\
\text { territory }(S)\end{array}$ & $\begin{array}{l}\text { Wounds in the } \\
\text { body (roots) }\end{array}$ & $\begin{array}{l}\text { Medicinal wine - } \\
\text { ingestion }\end{array}$ & $\begin{array}{l}\text { Antimalarial activity [119]and antifungal } \\
\text { activity [120] }\end{array}$ \\
\hline $\begin{array}{l}\text { 26-Cavalinha } \\
(\mathrm{MG} 1)^{\square}\end{array}$ & $\begin{array}{l}\text { Equisetum arvensis L. } \\
\text { (Equisetaceae) } \\
\text { Garcia } 051\end{array}$ & Exotic (C) & Diuretic (leaves) & $\begin{array}{l}\text { Infusion - } \\
\text { ingestion }\end{array}$ & No data found \\
\hline $\begin{array}{l}\text { 27-Cipó-cruz } \\
(\mathrm{SE} 1, \mathrm{PE} 2)^{\triangle}\end{array}$ & $\begin{array}{l}\text { Serjania sp. } \\
\text { (Sapindaceae) } \\
\text { Garcia } 012\end{array}$ & No data (S) & $\begin{array}{l}\text { Reduces } \\
\text { cholesterol and } \\
\text { diarrhea (leaves) }\end{array}$ & $\begin{array}{l}\text { Macerate - } \\
\text { ingestion }\end{array}$ & Not consulted \\
\hline
\end{tabular}


Table 2: The 78 plant specimens used by five Diadema's migrants (MG1, SP1, PE1, PE2, SE1)*. (Continued)

\begin{tabular}{|c|c|c|c|c|c|}
\hline & & & $\begin{array}{l}\text { External allergies, } \\
\text { wounds in the } \\
\text { body and } \\
\text { detoxifying } \\
\text { (leaves) }\end{array}$ & Infusion - bath & \\
\hline $\begin{array}{l}\text { 28-Comigo- } \\
\text { ninguém-pode } \\
(\mathrm{PE} 1)^{\square}\end{array}$ & $\begin{array}{l}\text { Dieffenbachia sp. } \\
\text { (Araceae) } \\
\text { Garcia } 071\end{array}$ & No data $(C)$ & $\begin{array}{l}\text { Toxic (whole } \\
\text { plant) }\end{array}$ & $\begin{array}{l}\text { Any oral dose is } \\
\text { dangerous }\end{array}$ & Not consulted \\
\hline $\begin{array}{l}\text { 29-Dormideira } \\
(\mathrm{SE} 1)^{\square}\end{array}$ & $\begin{array}{l}\text { Mimosa pudica L. } \\
\text { (Fabaceae s.I.) } \\
\text { Garcia } 069\end{array}$ & $\begin{array}{l}\text { Exotic - Brazilian } \\
\text { territory }(C)\end{array}$ & $\begin{array}{l}\text { Healing wounds } \\
\text { (aerial parts) }\end{array}$ & $\begin{array}{l}\text { In natura - } \\
\text { plaster }\end{array}$ & $\begin{array}{l}\text { Antidepressant activity [121], antitoxin } \\
\text { of the snake Naja kaouthia [122], } \\
\text { anticonvulsant [123]and for } \\
\text { reproductive problems [124] }\end{array}$ \\
\hline \multirow[t]{2}{*}{$\begin{array}{l}\text { 30-Embaúba } \\
(\mathrm{MG} 1, \mathrm{SE} 1)^{\square}\end{array}$} & $\begin{array}{l}\text { Cecropia pachystachya } \\
\text { Tréc. (Cecropiaceae) } \\
\text { Garcia } 068\end{array}$ & $\begin{array}{l}\text { Native - south to } \\
\text { northeast Brazil (S) }\end{array}$ & $\begin{array}{l}\text { Bronchitis* } \\
\text { (powder fruit) }\end{array}$ & Syrup - ingestion & $\begin{array}{l}\text { Antioxidative activity [125], cardiotonic } \\
\text { and sedative effects [126]and anti- } \\
\text { inflammatory }[127]\end{array}$ \\
\hline & & & Toxic (sap) & $\begin{array}{l}\text { Any oral dose is } \\
\text { dangerous }\end{array}$ & \\
\hline \multirow[t]{2}{*}{$\begin{array}{l}\text { 31-Erva-cidreira } \\
(M G 1, S E 1, P E 2)^{\square}\end{array}$} & $\begin{array}{l}\text { Lippia alba (Mill.) N. E. } \\
\text { Br. (Verbenaceae) } \\
\text { Garcia } 005\end{array}$ & $\begin{array}{l}\text { Native - almost all } \\
\text { Brazilian territory (S) }\end{array}$ & $\begin{array}{l}\text { Expectorant* } \\
\text { (aerial parts) }\end{array}$ & $\begin{array}{l}\text { Infusion - } \\
\text { inhalation }\end{array}$ & $\begin{array}{l}\text { Treatment of respiratory diseases [128], } \\
\text { antiulcerogenic activity [129], sedative } \\
\text { and anticonvulsant effects [130], } \\
\text { antiviral and antiherpes [131] }\end{array}$ \\
\hline & & & $\begin{array}{l}\text { Sedative* (aerial } \\
\text { parts) }\end{array}$ & $\begin{array}{l}\text { Infusion or } \\
\text { decoction - } \\
\text { ingestion }\end{array}$ & \\
\hline $\begin{array}{l}\text { 32-Erva-de-bicho } \\
(\mathrm{SE} 1)^{\square}\end{array}$ & $\begin{array}{l}\text { Ludwigia sp. } \\
\text { (Onagraceae) } \\
\text { Garcia } 078\end{array}$ & No data (S) & $\begin{array}{l}\text { Hemorrhoid } \\
\text { (whole plant) }\end{array}$ & Decoction - bath & Not consulted \\
\hline \multirow[t]{3}{*}{$\begin{array}{l}\text { 33-Erva-doce, } \\
\text { funcho (MG1, } \\
\text { SP1, PE1, PE2) }\end{array}$} & $\begin{array}{l}\text { Foeniculum vulgare } \\
\text { Mill. (Apiaceae) } \\
\text { Garcia } 064\end{array}$ & $\begin{array}{l}\text { Exotic -Brazilian } \\
\text { territory }(C)\end{array}$ & $\begin{array}{l}\text { Sedative (whole } \\
\text { plant) }\end{array}$ & $\begin{array}{l}\text { Infusion - } \\
\text { ingestion }\end{array}$ & $\begin{array}{l}\text { Antimicrobial activity [132], anti- } \\
\text { inflammatory, analgesic and } \\
\text { antioxidant activities [133], acaricidal } \\
\text { activity [134], antifungal effect [135], } \\
\text { antithrombotic activity [136]and } \\
\text { larvicidal activity of the mosquito Aedes } \\
\text { aegypti [137] }\end{array}$ \\
\hline & & & $\begin{array}{l}\text { Bronchitis* (whole } \\
\text { plant) }\end{array}$ & $\begin{array}{l}\text { Infusion - } \\
\text { inhalation }\end{array}$ & \\
\hline & & & $\begin{array}{l}\text { Laxative (whole } \\
\text { plant) }\end{array}$ & $\begin{array}{l}\text { Infusion or } \\
\text { macerate - } \\
\text { ingestion }\end{array}$ & \\
\hline $\begin{array}{l}\text { 34-Eucalipto, vick } \\
\left(\mathrm{MG} 1^{\square}, \mathrm{PE} 1^{\triangle}\right. \\
\left.\mathrm{PE}^{\Delta}, \mathrm{SE}^{\square}\right)\end{array}$ & $\begin{array}{l}\text { Eucalyptus globulus } \\
\text { Labill. (Myrtaceae) } \\
\text { Garcia } 055\end{array}$ & Exotic (C) & Sinusitis* (leaves) & $\begin{array}{l}\text { Infusion - } \\
\text { inhalation }\end{array}$ & $\begin{array}{l}\text { Antihyperglycemic actions [138], } \\
\text { analgesic and anti-inflammatory effects } \\
\text { [139], antimicrobial activity [140]and } \\
\text { antibacterial effects [141] }\end{array}$ \\
\hline $\begin{array}{l}\text { 35-Fedegoso } \\
(\mathrm{MG} 1)^{\square}\end{array}$ & $\begin{array}{l}\text { Senna pendula } \\
\text { (Humb. \& Bonpl. ex } \\
\text { Willd.) H.S. Irwin \& } \\
\text { Barneby (Fabaceae s. } \\
\text { I.) } \\
\text { Garcia } 034\end{array}$ & $\begin{array}{l}\text { Native - Brazilian } \\
\text { territory (S) }\end{array}$ & $\begin{array}{l}\text { Osteoporosis } \\
\text { prevention (roots) }\end{array}$ & $\begin{array}{l}\text { Medicinal wine - } \\
\text { ingestion }\end{array}$ & No data found \\
\hline $\begin{array}{l}\text { 36-Feijão-guandu } \\
(\mathrm{SP} 1)^{\square}\end{array}$ & $\begin{array}{l}\text { Cajanus cajan (L.) } \\
\text { Millsp. (Fabaceae s.I.) } \\
\text { Garcia } 003\end{array}$ & $\begin{array}{l}\text { Exotic - Brazilian } \\
\text { territory }(\mathrm{C})\end{array}$ & Bronchitis (leaves) & $\begin{array}{l}\text { Infusion - } \\
\text { ingestion or } \\
\text { inhalation }\end{array}$ & $\begin{array}{l}\text { Treatment of postmenopausal } \\
\text { osteoporosis [142], antileishmanial and } \\
\text { antifungal activity [143]and } \\
\text { hypocholesterolemic effect [144] }\end{array}$ \\
\hline \multirow[t]{2}{*}{$\begin{array}{l}\text { 37-Folha-santa, } \\
\text { folha-da-fortuna } \\
(\mathrm{MG} 1, \mathrm{SP} 1, \mathrm{PE} 1)^{\square}\end{array}$} & $\begin{array}{l}\text { Bryophyllum pinnatum } \\
\text { (Lam.) Oken } \\
\text { (Crassulaceae) } \\
\text { Garcia } 040\end{array}$ & $\begin{array}{l}\text { Exotic - Brazilian } \\
\text { territory }(C)\end{array}$ & $\begin{array}{l}\text { Lumbar pain* } \\
\text { (leaves) }\end{array}$ & $\begin{array}{l}\text { In natura - } \\
\text { plaster }\end{array}$ & $\begin{array}{l}\text { Antibacterial activity [145], anti-ulcer } \\
\text { [146], antimicrobial [147], } \\
\text { antinociceptive, anti-inflammatory and } \\
\text { antidiabetic [148] and neurosedative and } \\
\text { muscle relaxant activities [149] }\end{array}$ \\
\hline & & & Sedative* (leaves) & $\begin{array}{l}\text { In natura - } \\
\text { plaster }\end{array}$ & \\
\hline
\end{tabular}


Table 2: The 78 plant specimens used by five Diadema's migrants (MG1, SP1, PE1, PE2, SE1)*. (Continued)

\begin{tabular}{|c|c|c|c|c|c|}
\hline $\begin{array}{l}\text { 38-Gervão } \\
(\mathrm{MG} 1)^{\square}\end{array}$ & $\begin{array}{l}\text { Stachytarpheta } \\
\text { cayennensis (Rich.) } \\
\text { Vahl (Verbenaceae) } \\
\text { Garcia } 054\end{array}$ & $\begin{array}{l}\text { Native - Brazilian } \\
\text { territory }(S)\end{array}$ & $\begin{array}{l}\text { Laxative (aerial } \\
\text { parts) }\end{array}$ & $\begin{array}{l}\text { Infusion or } \\
\text { decoction - } \\
\text { ingestion }\end{array}$ & $\begin{array}{l}\text { Anti-inflammatory and anti-ulcerogenic } \\
\text { properties }[150] \text { and hypoglycaemic } \\
\text { constituents }[151]\end{array}$ \\
\hline \multirow[t]{2}{*}{ 39-Goiaba (SE1) } & $\begin{array}{l}\text { Psidium guajava L. } \\
\text { (Myrtaceae) } \\
\text { Garcia } 058\end{array}$ & $\begin{array}{l}\text { Native - Mexico to } \\
\text { Brazil (S) }\end{array}$ & Heartburn (leaves) & $\begin{array}{l}\text { Infusion or in } \\
\text { natura - } \\
\text { ingestion }\end{array}$ & $\begin{array}{l}\text { Antibacterial activity }[152-154] \text { and } \\
\text { hepatoprotective activity }[155]\end{array}$ \\
\hline & & & Diarrhea (fruit) & $\begin{array}{l}\text { In natura - } \\
\text { ingestion }\end{array}$ & \\
\hline $\begin{array}{l}\text { 40-Guaco }\left(P E 1^{\square}\right. \\
\left.\mathrm{PE}^{\square}, \mathrm{SE}^{\Delta}{ }^{\Delta}\right)\end{array}$ & $\begin{array}{l}\text { Mikania glomerata } \\
\text { Spreng. (Asteraceae) } \\
\text { Garcia } 032\end{array}$ & $\begin{array}{l}\text { Native - northeast to } \\
\text { southeast Brazil (S) }\end{array}$ & $\begin{array}{l}\text { Bronchitis* } \\
\text { (leaves) }\end{array}$ & Syrup - ingestion & $\begin{array}{l}\text { Analgesic and anti-inflammatory } \\
\text { activities [156], bronchodilator activity } \\
\text { [157]and antiophidian properties [158] }\end{array}$ \\
\hline $\begin{array}{l}\text { 41-Guanxuma } \\
(\mathrm{SE} 1)^{\triangle}\end{array}$ & $\begin{array}{l}\text { Sida rhombifolia L. } \\
\text { (Malvaceae) } \\
\text { Garcia } 067\end{array}$ & $\begin{array}{l}\text { Exotic - Brazilian } \\
\text { territory }(S)\end{array}$ & $\begin{array}{l}\text { Sedative (aerial } \\
\text { parts) }\end{array}$ & $\begin{array}{l}\text { Infusion - } \\
\text { ingestion or } \\
\text { inhalation }\end{array}$ & $\begin{array}{l}\text { Cytotoxicity, antibacterial activity [159] } \\
\text { and antioxidant [160] }\end{array}$ \\
\hline \multirow[t]{2}{*}{ 42-Guiné (SE1) } & $\begin{array}{l}\text { Petiveria alliaceae L. } \\
\text { (Phytolaccaceae) } \\
\text { Garcia } 004\end{array}$ & $\begin{array}{l}\text { Native - north Brazil } \\
\text { (S) }\end{array}$ & $\begin{array}{l}\text { Sedative (aerial } \\
\text { parts) }\end{array}$ & $\begin{array}{l}\text { Environment } \\
\text { purifier - } \\
\text { inhalation }\end{array}$ & $\begin{array}{l}\text { Antimicrobial substance [161], } \\
\text { antimitotic action [162], anti- } \\
\text { inflammatory and analgesic effects } \\
\text { [163], antibacterial and antifungal } \\
\text { activity [164]and antioxidant [165] }\end{array}$ \\
\hline & & & $\begin{array}{l}\text { Muscle pain* } \\
\text { (leaves) }\end{array}$ & $\begin{array}{l}\text { Decoction - } \\
\text { massage }\end{array}$ & \\
\hline \multirow[t]{2}{*}{$\begin{array}{l}\text { 43-Hortelã (MG1, } \\
\text { PE1) }\end{array}$} & $\begin{array}{l}\text { Mentha arvensis L. } \\
\text { (Lamiaceae) } \\
\text { Garcia } 031\end{array}$ & $\begin{array}{l}\text { Exotic - Brazilian } \\
\text { territory }(C)\end{array}$ & $\begin{array}{l}\text { Bronchitis* } \\
\text { (leaves) }\end{array}$ & Syrup - ingestion & $\begin{array}{l}\text { Antifungal property [166], vasodilatory } \\
\text { actions [167], antioxidative activity [168], } \\
\text { antibacterial properties [107]and insect } \\
\text { repellents and fumigants [109] }\end{array}$ \\
\hline & & & Laxative (leaves) & $\begin{array}{l}\text { Infusion - } \\
\text { ingestion }\end{array}$ & \\
\hline \multirow[t]{2}{*}{$\begin{array}{l}\text { 44-Hortelã- } \\
\text { grande }(P E 1)^{\square}\end{array}$} & $\begin{array}{l}\text { Plectranthus } \\
\text { amboinicus (Lour.) } \\
\text { Spreng. (Lamiaceae) } \\
\text { Garcia } 073\end{array}$ & $\begin{array}{l}\text { Exotic - Brazilian } \\
\text { territory }(C)\end{array}$ & $\begin{array}{l}\text { For digestion and } \\
\text { urine with blood } \\
\text { (leaves) }\end{array}$ & $\begin{array}{l}\text { Infusion - } \\
\text { ingestion }\end{array}$ & $\begin{array}{l}\text { Scorpion venon antidote [169]and } \\
\text { antimicrobial activity }[92]\end{array}$ \\
\hline & & & Cough (leaves) & Syrup - ingestion & \\
\hline $\begin{array}{l}\text { 45-Impatiens } \\
(\mathrm{PE} 1)^{\triangle}\end{array}$ & $\begin{array}{l}\text { Impatiens hawkeri W. } \\
\text { Bull. (Balsaminaceae) } \\
\text { Garcia } 044\end{array}$ & $\begin{array}{l}\text { Exotic - Brazilian } \\
\text { territory }(C)\end{array}$ & $\begin{array}{l}\text { Toxic (whole } \\
\text { plant) }\end{array}$ & $\begin{array}{l}\text { In closed } \\
\text { environment } \\
\text { causes tearing, } \\
\text { allergy and } \\
\text { headache }\end{array}$ & No data found \\
\hline \multirow[t]{2}{*}{$\begin{array}{l}\text { 46-Jarnaúba } \\
(\mathrm{PE} 1)^{\triangle}\end{array}$} & $\begin{array}{l}\text { Synadenium grantii } \\
\text { Hook. F. } \\
\text { (Euphorbiaceae) } \\
\text { Garcia } 074\end{array}$ & $\begin{array}{l}\text { Exotic - southeast to } \\
\text { northeast Brazil }(C)\end{array}$ & $\begin{array}{l}\text { Toxic (whole } \\
\text { plant) }\end{array}$ & Restricted use & $\begin{array}{l}\text { Healing action and anti-hemorrhagic } \\
{[170]}\end{array}$ \\
\hline & & & $\begin{array}{l}\text { Stomach cancer } \\
\text { (latex) }\end{array}$ & $\begin{array}{l}\text { Macerate - } \\
\text { ingestion }\end{array}$ & \\
\hline \multirow[t]{2}{*}{$\begin{array}{l}\text { 47-Jurubeba } \\
(\mathrm{MG} 1, \mathrm{SE} 1, \mathrm{PE} 2)^{\square}\end{array}$} & $\begin{array}{l}\text { Solanum variabile } \\
\text { Mart. (Solanaceae) } \\
\text { Garcia } 056\end{array}$ & $\begin{array}{l}\text { Native - southeast } \\
\text { and south Brazil (S) }\end{array}$ & Sedative (leaves) & $\begin{array}{l}\text { Infusion - } \\
\text { ingestion }\end{array}$ & Antiulcerogenic activity [171] \\
\hline & & & $\begin{array}{l}\text { Laxative (powder } \\
\text { fruit) }\end{array}$ & $\begin{array}{l}\text { In natura - } \\
\text { ingestion }\end{array}$ & \\
\hline 48-Limão $(M G 1)^{\square}$ & $\begin{array}{l}\text { Citrus aurantifolia } \\
\text { (Christm.) Swingle } \\
\text { (Rutaceae) } \\
\text { Garcia } 063\end{array}$ & $\begin{array}{l}\text { Exotic - Brazilian } \\
\text { territory }(\mathrm{C})\end{array}$ & Fever (leaves) & $\begin{array}{l}\text { Infusion - } \\
\text { ingestion }\end{array}$ & Mosquito repellent activity [172] \\
\hline $\begin{array}{l}\text { 49-Losna (SP1, } \\
\text { SE1, PE2) }\end{array}$ & $\begin{array}{l}\text { Artemisia absinthium } \\
\text { L. (Asteraceae) } \\
\text { Garcia } 049\end{array}$ & $\begin{array}{l}\text { Exotic - Brazilian } \\
\text { territory }(S)\end{array}$ & $\begin{array}{l}\text { Laxative (aerial } \\
\text { parts) }\end{array}$ & $\begin{array}{l}\text { Infusion - } \\
\text { ingestion }\end{array}$ & $\begin{array}{l}\text { Acaricidal properties [173], antifungal } \\
\text { and antibacterial [174]and antioxidant } \\
\text { activities [175] }\end{array}$ \\
\hline $\begin{array}{l}\text { 50-Malva-branca } \\
(\mathrm{SE} 1)^{\square}\end{array}$ & $\begin{array}{l}\text { Waltheria indica L. } \\
\text { (Sterculiaceae) } \\
\text { Garcia } 077\end{array}$ & $\begin{array}{l}\text { Native - Brazilian } \\
\text { territory }(\mathrm{S})\end{array}$ & Gingivitis* (leaves) & $\begin{array}{l}\text { Infusion - } \\
\text { gargling }\end{array}$ & Anti-inflammatory activities [176] \\
\hline
\end{tabular}


Table 2: The 78 plant specimens used by five Diadema's migrants (MG1, SP1, PE1, PE2, SE1)*. (Continued)

\begin{tabular}{|c|c|c|c|c|c|}
\hline & & & $\begin{array}{l}\text { Inflammation in } \\
\text { the mouth and/or } \\
\text { throat* (leaves) }\end{array}$ & & \\
\hline $\begin{array}{l}\text { 51-Malva-de- } \\
\text { cheiro (MG1) }\end{array}$ & $\begin{array}{l}\text { Malva sylvestris L. } \\
\text { (Malvaceae) } \\
\text { Garcia } 059\end{array}$ & $\begin{array}{l}\text { Exotic - south and } \\
\text { southeast Brazil (S) }\end{array}$ & $\begin{array}{l}\text { Wounds in the } \\
\text { body (roots) }\end{array}$ & $\begin{array}{l}\text { Medicinal wine - } \\
\text { ingestion }\end{array}$ & Skin anti-aging property [177] \\
\hline $\begin{array}{l}\text { 52-Mamão- } \\
\text { papaia (PE1) }\end{array}$ & $\begin{array}{l}\text { Carica papaya L. } \\
\text { (Caricaceae) } \\
\text { Garcia } 062\end{array}$ & $\begin{array}{l}\text { Exotic - Brazilian } \\
\text { territory }(C)\end{array}$ & $\begin{array}{l}\text { Bronchitis* } \\
\text { (powder fruit) }\end{array}$ & Syrup - ingestion & $\begin{array}{l}\text { Abortive [178], antibacterial activity } \\
\text { [179], diuretic [180]and healing and } \\
\text { abortive effects [181] }\end{array}$ \\
\hline $\begin{array}{l}\text { 53-Mandioca } \\
(\mathrm{SE} 1)^{\square}\end{array}$ & $\begin{array}{l}\text { Manihot esculenta } \\
\text { Crantz } \\
\text { (Euphorbiaceae) } \\
\text { Garcia } 050\end{array}$ & $\begin{array}{l}\text { Native - Brazilian } \\
\text { territory (C) }\end{array}$ & $\begin{array}{l}\text { conjunctivitis/sty* } \\
\text { (dew on the } \\
\text { leaves) }\end{array}$ & In natura - topic & $\begin{array}{l}\text { Analgesics and anti-inflammatory } \\
\text { effects [182] }\end{array}$ \\
\hline $\begin{array}{l}\text { 54-Manjericão } \\
(\text { MG1 })^{\square}\end{array}$ & $\begin{array}{l}\text { Ocimum basilicum L. } \\
\text { (Lamiaceae) } \\
\text { Garcia } 061\end{array}$ & $\begin{array}{l}\text { Exotic - Brazilian } \\
\text { territory }(C)\end{array}$ & $\begin{array}{l}\text { Bronchitis* } \\
\text { (leaves) }\end{array}$ & Syrup - ingestion & $\begin{array}{l}\text { Antibacterial [183], mosquito repellent } \\
\text { activity [184], antimicrobial activity [185] } \\
\text { antigiardial activity [186]and decreases } \\
\text { cholesterol [187] }\end{array}$ \\
\hline $\begin{array}{l}\text { 55-Maravilha } \\
(\mathrm{SP} 1, \mathrm{PE} 2)^{口}\end{array}$ & $\begin{array}{l}\text { Mirabilis jalapa L. } \\
\text { (Nyctaginaceae) } \\
\text { Garcia } 065\end{array}$ & $\begin{array}{l}\text { Native - Brazilian } \\
\text { territory (C) }\end{array}$ & $\begin{array}{l}\text { Healing wounds* } \\
\text { (aerial parts) }\end{array}$ & Infusion - plaster & $\begin{array}{l}\text { Antibacterial effect [188]and } \\
\text { antimicrobial [189] }\end{array}$ \\
\hline $\begin{array}{l}\text { 56-Maria- } \\
\text { pretinha }(M G 1)^{\square}\end{array}$ & $\begin{array}{l}\text { Solanum americanum } \\
\text { L. (Solanaceae) } \\
\text { Garcia } 070\end{array}$ & Native - Americas (S) & $\begin{array}{l}\text { Sore throat* } \\
\text { (aerial parts) }\end{array}$ & Infusion - gargle & $\begin{array}{l}\text { Treatment of protozoal infections } \\
\text { (American trypanosomes) [190]and } \\
\text { moderate antioxidant activity [191] }\end{array}$ \\
\hline \multirow[t]{2}{*}{$\begin{array}{l}\text { 57-Mentrasto } \\
(\mathrm{PE} 1)^{\square}\end{array}$} & $\begin{array}{l}\text { Ageratum conyzoides } \\
\text { L. (Asteraceae) } \\
\text { Garcia } 010\end{array}$ & $\begin{array}{l}\text { Native - southeast to } \\
\text { northeast Brazil } \\
\text { (S) }\end{array}$ & $\begin{array}{l}\text { Bronchitis* } \\
\text { (leaves) }\end{array}$ & $\begin{array}{l}\text { Infusion - } \\
\text { ingestion }\end{array}$ & $\begin{array}{l}\text { Anti-inflammatory [192], toxic [193], } \\
\text { antibacterial [194]and insecticidal } \\
\text { activity [195] }\end{array}$ \\
\hline & & & $\begin{array}{l}\text { Rheumatism* } \\
\text { (whole plant) }\end{array}$ & Infusion - bath & \\
\hline \multirow[t]{4}{*}{$\begin{array}{l}\text { 58-Mentruz, erva- } \\
\text { de-santa-maria } \\
\left(\mathrm{PE1}^{\#}, \mathrm{SE1} 1^{\square}\right)\end{array}$} & $\begin{array}{l}\text { Chenopodium } \\
\text { ambrosioides L. } \\
\text { (Chenopodiaceae) } \\
\text { Garcia } 006\end{array}$ & $\begin{array}{l}\text { Native - south and } \\
\text { southeast Brazil (S) }\end{array}$ & $\begin{array}{l}\text { Muscle pain } \\
\text { (aerial parts) }\end{array}$ & $\begin{array}{l}\text { Decoction - } \\
\text { massage }\end{array}$ & $\begin{array}{l}\text { Insecticidal properties [196], antifungal, } \\
\text { antiaflatoxigenic and antioxidant } \\
\text { activity [197] and mosquito repellent } \\
\text { activity [71] }\end{array}$ \\
\hline & & & $\begin{array}{l}\text { Lesions in bone } \\
\text { (aerial parts) }\end{array}$ & $\begin{array}{l}\text { In natura - } \\
\text { plaster }\end{array}$ & \\
\hline & & & $\begin{array}{l}\text { Worm* (aerial } \\
\text { parts) }\end{array}$ & $\begin{array}{l}\text { Infusion - } \\
\text { ingestion }\end{array}$ & \\
\hline & & & $\begin{array}{l}\text { Bronchitis (aerial } \\
\text { parts) }\end{array}$ & Syrup - ingestion & \\
\hline \multirow[t]{2}{*}{ 59-Milho (SE1) } & $\begin{array}{l}\text { Zea mays L. (Poaceae) } \\
\text { Garcia } 023\end{array}$ & $\begin{array}{l}\text { Exotic - Brazilian } \\
\text { territory (C) }\end{array}$ & $\begin{array}{l}\text { Bronchitis } \\
\text { (flowers) }\end{array}$ & Syrup - ingestion & No data found \\
\hline & & & $\begin{array}{l}\text { Blood purifier and } \\
\text { diuretic (flowers) }\end{array}$ & $\begin{array}{l}\text { Infusion - } \\
\text { ingestion }\end{array}$ & \\
\hline $\begin{array}{l}\text { 60-Novalgina } \\
(\mathrm{MG} 1, \mathrm{SE} 1)^{\square}\end{array}$ & $\begin{array}{l}\text { Achillea millefolium L. } \\
\text { (Asteraceae) } \\
\text { Garcia } 015\end{array}$ & $\begin{array}{l}\text { Exotic - south and } \\
\text { southeast Brazil (C) }\end{array}$ & Sedative (leaves) & $\begin{array}{l}\text { In natura - } \\
\text { ingestion }\end{array}$ & $\begin{array}{l}\text { Antioxidant and antimicrobial activity } \\
\text { [198] }\end{array}$ \\
\hline $\begin{array}{l}\text { 61-Pariparoba } \\
(\mathrm{MG} 1)^{\square}\end{array}$ & $\begin{array}{l}\text { Piper umbellatum L. } \\
\text { (Piperaceae) } \\
\text { Garcia } 072\end{array}$ & $\begin{array}{l}\text { Native - Tropical } \\
\text { America (S) }\end{array}$ & $\begin{array}{l}\text { Belly ache and } \\
\text { liver pain (leaves) }\end{array}$ & $\begin{array}{l}\text { Infusion - } \\
\text { ingestion }\end{array}$ & $\begin{array}{l}\text { Antioxidant [199] and antifungal activity } \\
\text { [200] }\end{array}$ \\
\hline 62-Picão (MG1) & $\begin{array}{l}\text { Calea sp. (Asteraceae) } \\
\text { Garcia } 036\end{array}$ & No data (S) & Diuretic (leaves) & $\begin{array}{l}\text { Infusion - } \\
\text { ingestion }\end{array}$ & Not consulted \\
\hline \multirow[t]{3}{*}{$\begin{array}{l}\text { 63-Picão-preto } \\
\text { (MG1, PE1) } \\
\text { Picão-branco } \\
\text { (SP1) }\end{array}$} & $\begin{array}{l}\text { Bidens pilosa L. } \\
\text { (Asteraceae) } \\
\text { Garcia } 020\end{array}$ & $\begin{array}{l}\text { Native - tropical } \\
\text { America (S) }\end{array}$ & $\begin{array}{l}\text { Blood purifier } \\
\text { (whole plant) }\end{array}$ & $\begin{array}{l}\text { Infusion - } \\
\text { ingestion }\end{array}$ & $\begin{array}{l}\text { Hypotensive effects [201], anti- } \\
\text { inflammatory activity [202], anticancer } \\
\text { and antipyretic activity [203], } \\
\text { antimicrobial [204]and antitumor } \\
\text { potential [205] }\end{array}$ \\
\hline & & & $\begin{array}{l}\text { Healing wounds* } \\
\text { (whole plant) }\end{array}$ & $\begin{array}{l}\text { In natura - } \\
\text { plaster }\end{array}$ & \\
\hline & & & $\begin{array}{l}\text { Wounds in the } \\
\text { body* (roots) }^{*}\end{array}$ & $\begin{array}{l}\text { Medicinal wine - } \\
\text { ingestion }\end{array}$ & \\
\hline
\end{tabular}


Table 2: The 78 plant specimens used by five Diadema's migrants (MG1, SP1, PE1, PE2, SE1)*. (Continued)

\begin{tabular}{|c|c|c|c|c|c|}
\hline $\begin{array}{l}\text { 64-Pinhão-roxo } \\
\text { (SP1) }\end{array}$ & $\begin{array}{l}\text { Jatropha gossypiifolia } \\
\text { L. (Euphorbiaceae) } \\
\text { Garcia } 017\end{array}$ & $\begin{array}{l}\text { Native - southeast to } \\
\text { northeast Brazil (S) }\end{array}$ & $\begin{array}{l}\text { Laxative (powder } \\
\text { fruit) }\end{array}$ & $\begin{array}{l}\text { In natura - } \\
\text { ingestion }\end{array}$ & $\begin{array}{l}\text { Antimalarial effects [206], hypotensive } \\
\text { and vasorelaxant effects [207] }\end{array}$ \\
\hline $\begin{array}{l}\text { 65-Poejo (MG1, } \\
\text { PE2) }\end{array}$ & $\begin{array}{l}\text { Mentha pulegium L. } \\
\text { (Lamiaceae) } \\
\text { Garcia } 029\end{array}$ & $\begin{array}{l}\text { Exotic - Brazilian } \\
\text { territory }(C)\end{array}$ & Bronchitis (leaves) & Syrup - ingestion & $\begin{array}{l}\text { Larvicidal activity [208], acaricidal effects } \\
\text { [209] and insecticidal properties [210] }\end{array}$ \\
\hline$\overline{66-P u c u n a ̃ ~(S E 1) ~}$ & $\begin{array}{l}\text { Fevillea passiflora Vell. } \\
\text { (Cucurbitaceae) } \\
\text { Garcia } 022\end{array}$ & $\begin{array}{l}\text { Native - North and } \\
\text { southeast Brazil (S) }\end{array}$ & $\begin{array}{l}\text { Toxic - abortive } \\
\text { (seeds) }\end{array}$ & $\begin{array}{l}\text { In natura - } \\
\text { ingestion }\end{array}$ & No data found \\
\hline $\begin{array}{l}\text { 67-Quebra-pedra } \\
\text { (SP1, PE1, PE2, } \\
\text { SE1) }\end{array}$ & $\begin{array}{l}\text { Phyllanthus } \\
\text { caroliniensis Walter } \\
\text { (Euphorbiaceae) } \\
\text { Garcia } 024\end{array}$ & $\begin{array}{l}\text { Native - USA to Brazil } \\
\text { (S) }\end{array}$ & $\begin{array}{l}\text { Kidney stone* } \\
\text { (aerial parts) }\end{array}$ & $\begin{array}{l}\text { Infusion or } \\
\text { decoction - } \\
\text { ingestion }\end{array}$ & Antinociceptive action [211] \\
\hline $\begin{array}{l}\text { 68-Quitoco } \\
(\mathrm{SE} 1)^{\square}\end{array}$ & $\begin{array}{l}\text { Pluchea sagittalis } \\
\text { (Lam.) Cabrera } \\
\text { (Asteraceae) } \\
\text { Garcia } 042 \\
\end{array}$ & $\begin{array}{l}\text { Native - south and } \\
\text { southeast Brazil (S) }\end{array}$ & $\begin{array}{l}\text { Diuretic (aerial } \\
\text { parts) }\end{array}$ & $\begin{array}{l}\text { Infusion - } \\
\text { ingestion }\end{array}$ & Anti-inflammatory activity [212] \\
\hline $\begin{array}{l}\text { 69-Rubim (MG1, } \\
\text { SP1) }\end{array}$ & $\begin{array}{l}\text { Leonurus sibiricus L. } \\
\text { (Lamiaceae) } \\
\text { Garcia } 002\end{array}$ & $\begin{array}{l}\text { Exotic - Brazilian } \\
\text { territory }(C)\end{array}$ & $\begin{array}{l}\text { Healing wounds* } \\
\text { (aerial parts) }\end{array}$ & $\begin{array}{l}\text { In natura - } \\
\text { plaster }\end{array}$ & $\begin{array}{l}\text { Stimulating action on the uterus }[213], \\
\text { analgesic and anti-inflammatory activity } \\
{[214] \text { and antibacterial activity }[215]}\end{array}$ \\
\hline $\begin{array}{l}\text { 70-Sabugueiro } \\
\text { (MG1) }\end{array}$ & $\begin{array}{l}\text { Sambucus canadensis } \\
\text { L. (Caprifoliaceae) } \\
\text { Garcia } 025\end{array}$ & $\begin{array}{l}\text { Native - Brazilian } \\
\text { territory }(S)\end{array}$ & $\begin{array}{l}\text { Bronchitis* } \\
\text { (flowers) }\end{array}$ & Syrup - ingestion & $\begin{array}{l}\text { Infectious diseases and antioxidant } \\
\text { activity [216] }\end{array}$ \\
\hline $\begin{array}{l}\text { 71-Salsa-parreira } \\
(\mathrm{SE} 1)^{\square}\end{array}$ & $\begin{array}{l}\text { Jacaranda sp. } \\
\text { (Bignoniaceae) } \\
\text { Garcia } 011\end{array}$ & No data (S) & $\begin{array}{l}\text { External allergies, } \\
\text { wounds in the } \\
\text { body and purifier } \\
\text { (leaves) }\end{array}$ & Decoction - bath & Not consulted \\
\hline $\begin{array}{l}\text { 72-Samba-caitá } \\
(\text { SE1 })^{\square}\end{array}$ & $\begin{array}{l}\text { Hyptis sp. (Lamiaceae) } \\
\text { Garcia } 041\end{array}$ & No data (S) & Belly ache (leaves) & $\begin{array}{l}\text { In natura - } \\
\text { ingestion }\end{array}$ & Not consulted \\
\hline $\begin{array}{l}\text { 73-Serralha } \\
(\mathrm{PE} 1)^{\square}\end{array}$ & $\begin{array}{l}\text { Sonchus oleraceus L. } \\
\text { (Asteraceae) } \\
\text { Garcia } 016\end{array}$ & $\begin{array}{l}\text { Exotic - Brazilian } \\
\text { territory }(S)\end{array}$ & Diabetes (leaves) & $\begin{array}{l}\text { In natura - } \\
\text { ingestion }\end{array}$ & Larvicidal potential [217] \\
\hline $\begin{array}{l}\text { 74-Sete-sangria } \\
\left(\mathrm{MG} 1^{\square}, \mathrm{SP} 1^{\square}\right. \\
\left.\mathrm{SE} 1^{\Delta}\right)\end{array}$ & $\begin{array}{l}\text { Cuphea carthagenensis } \\
\text { (Jacq.) J. F. Macbr. } \\
\text { (Lythraceae) } \\
\text { Garcia } 007\end{array}$ & $\begin{array}{l}\text { Native - Brazilian } \\
\text { territory (S) }\end{array}$ & $\begin{array}{l}\text { Intestinal } \\
\text { infections and } \\
\text { heart problems* } \\
\text { (aerial parts) }\end{array}$ & $\begin{array}{l}\text { Infusion - } \\
\text { ingestion }\end{array}$ & $\begin{array}{l}\text { Antiinflammatory and antinociceptive } \\
\text { activities [218], vasorelaxant properties } \\
\text { [219], treat high levels of cholesterol } \\
\text { and triglycerides [220] }\end{array}$ \\
\hline $\begin{array}{l}\text { 75-Sofre-do-rim- } \\
\text { quem-qué (MG1) }\end{array}$ & $\begin{array}{l}\text { Cissus sp. (Vitaceae) } \\
\text { Garcia } 053\end{array}$ & No data (S) & $\begin{array}{l}\text { Kidney stone } \\
\text { (leaves) }\end{array}$ & $\begin{array}{l}\text { Infusion - } \\
\text { ingestion }\end{array}$ & Not consulted \\
\hline $\begin{array}{l}\text { 76-Tanchagem } \\
(\text { SP1, PE2) }\end{array}$ & $\begin{array}{l}\text { Plantago sp. } \\
\text { (Plantaginaceae) } \\
\text { Garcia } 008\end{array}$ & No data (S) & $\begin{array}{l}\text { Anti-inflammatory } \\
\text { - mouth and } \\
\text { throat (leaves) }\end{array}$ & $\begin{array}{l}\text { Decoction - } \\
\text { gargling }\end{array}$ & Not consulted \\
\hline $\begin{array}{l}\text { 77-Vassourinha } \\
\text { (SE1, PE2) })^{\square}\end{array}$ & $\begin{array}{l}\text { Scoparia dulcis L. } \\
\text { (Scrophulariaceae) } \\
\text { Garcia } 014\end{array}$ & $\begin{array}{l}\text { Native - Brazilian } \\
\text { territory (S) }\end{array}$ & $\begin{array}{l}\text { Hip pain/kidneys } \\
\text { (leaves) }\end{array}$ & Decoction - bath & $\begin{array}{l}\text { Antitumor-promoting activity }[221], \\
\text { antioxidant }[222] \text {, antimicrobial and } \\
\text { antifungal activities [223] }\end{array}$ \\
\hline $\begin{array}{l}\text { 78-Velando } \\
(\mathrm{SE} 1)^{\square}\end{array}$ & $\begin{array}{l}\text { Croton fuscescens } \\
\text { Spreng } \\
\text { (Euphorbiaceae) } \\
\text { Garcia } 013\end{array}$ & $\begin{array}{l}\text { Native - Brazilian } \\
\text { territory }(S)\end{array}$ & $\begin{array}{l}\text { Inhibits the } \\
\text { growth of skin } \\
\text { stains/wounds in } \\
\text { the body (resin) }\end{array}$ & In natura - topic & No data found \\
\hline
\end{tabular}

* their popular and scientific names, geographical origin and distribution, if cultivated or spontaneous, uses, parts utilized, formula, route of administration and pharmacological studies. Marked by $\left(^{\square}\right)$ the 68 plants whose use had been maintained by the respective migrant, while 14 , marked by $\left(^{\Delta}\right)$ are those whose applications have been incorporated by migrants, finally, $3\left(^{\#}\right)$ are replacements. The matches between the uses proclaimed by the interviewees and pharmacological data have been posted by $\left(^{*}\right)$.

(highlighted with $\square$ in Table 2). The maintenance of their uses was possible since most of them were available in Diadema, though some were brought from their homelands. SE1 brought four plants from Aquidabã Sergipe state, for pain relief because they are not available or are more potent than the ones found in
Diadema: "bálsamo" (Sedum sp.), “anador" (Alternanthera sp.), "eucalipto/vick" (Eucalyptus globulus Labill.) and "novalgina" (Achillea millefolium L.).

\section{Incorporation of use}

Fourteen of the 78 specimens listed in Table 2 came to be used by migrants when they arrived in Diadema 
(highlighted with $\Delta$ in Table 2). These incorporations occurred in several ways: through information given by neighbours; through local media, e.g., television, radio, magazines; or through personal efforts, guided by plant organoleptic properties or even by the theory of signatures. This theory, formulated by Paracelsus (XVI century), assumes that characteristics and virtues of herbs can be recognised by their external appearance or "signature" (picture, shape, colour). Finally, observing the relationship between animals and plants can be a valuable guide. PE1 noted that dogs consume "sete-sangria" (Cuphea carthagenensis (Jacq.) J. F. Macbr.) when they have diarrhoea; and because it seemed to alleviate their symptoms, he started to use this plant for the same purpose.

The migrants incorporated several plants after their arrival in Diadema to treat typical diseases of larger cities: "cipó-cruz" (Serjania sp.) to combat high cholesterol; and "guanxuma" (Sida rhombifolia L.) and "guiné" (Petiveria alliaceae L.) for anxiety. Also included in this category was knowledge concerning local toxic plants, e. g., alamanda-amarela (Allamanda cathartica L.) and azaléia (Rhododendron simsii Planch.), detailing the risks associated with their consumption.

\section{Replacement of use}

Three plants used by migrants in their cities of origin were replaced because they were not available or were less effective than plants present in Diadema (highlighted with \# in Table 2). Most of these replacements were made according to the criteria listed in the previous section.

The interviewee MG1 explained that in his homelands, he used "quebra-pedra" (Phyllanthus cf. caroliniensis Walter - Euphorbiaceae) for kidney stone disturbance, but when he arrived in Diadema, he found another plant, "sofre-do-rim-quem-qué" (Cissus sp.), that seemed to have a stronger effect.

Another interviewee, PE1, reported that the bark and seeds of "amburana-de-cheiro": (Amburana cf. cearensis (Allemão) A.C. Sm. - Fabaceae s.l.) were widely used for anti-inflammatory therapy in Pernambuco state but had to be replaced by "mentruz" (Chenopodium ambrosioides L.) because the former was not found in Diadema. In addition, SE1 had to replace "pau-de-sapo"* (Pouteria cf. melinoniana Boehni - Sapotaceae), whose leaves were used for chronic wounds, with "carrapicho" (Acanthospermum australe (Loefl.) Kuntze).

The vernacular names of some plants are registered trademarks of allopathic medicines and active ingredients, e.g., Novalgina ${ }^{\circ}$ (Achillea millefolium) and Vick $^{\circ}$ (Eucalyptus globulus) for sinusitis, and Anador (Alternanthera sp.), which is used as a sedative and for general pain. Contact between migrants and allopathic medicine thus led to the 'baptisms' of these plants, following the observation that both, the commercially available products and herbal source have similar effects, as reported by Pires [43].

\section{Discontinued use}

According to MG1, the following plants used in his homeland fell into disuse because they were not found in Diadema, although he tried to acquire them from local commercial sources: "quina"* (Strychnos cf. pseudoquina A. St. Hil - Loganiaceae), whose root is used to combat pain in the stomach and intestine; bark oil of "jatobá":" (Hymenaea cf. courbaril L. - Fabaceae s.l.), used for combat wounds; "batata-de-purga"*: (Operculina cf. macrocarpa (L.) Urb - Convolvulaceae), whose tuber is ingested as a purgative and to clean the blood; bark and leaf of "jalapa"* (Mirabilis cf jalapa L. - Nyctaginaceae), used to clean the blood; tea of "junco": (Cyperus cf. esculentus L. - Cyperaceae), whose root is used for inflammation; bark or seed of "emburana"* (Amburana cf. cearensis - Fabaceae s.l.), used for migraine and sleeping; and bark of "angico":" (Anadenanthera cf. colubrina (Vell.) Brenan - Fabaceae s.l.), prepared as a tea for pain in the body and fever. These plants were not described in Table 2, since they could not be collected and identified as well.

\section{Plants used for therapeutic purposes}

Of the 78 plants, 10 carry some restrictions, as they can be toxic depending on the dose, route or part utilised (Table 2). The uses described in Table 2 are written just as they were reported by the interviewees. The 68 plants used exclusively for medicinal purposes were cited for 41 complaints, which were grouped into 12 functional categories according to bodily system, as detailed in Table 3. Thus, gastrointestinal disturbances include the following complaints (numbers of medicinal plants reported): endoparasitosis (1), ulcer (1), diarrhoea (1), bellyache (2), heartburn (1), intestinal infections (1), liver pain (3). This category also includes plants used to improve digestion (1), to treat tables of haemorrhoid (1), as laxatives (10) and to purify the stomach (2), comprising a total of 24 plants employed in 44 formulas.

The most relevant categories of use, measured by number of species employed, were gastrointestinal disturbances (30.8\% of plants), inflammatory processes $(24.4 \%)$ and respiratory problems $(23.1 \%)$. As seen in Table 4, the group of illnesses representing immunological problems obtained the highest informant consensus factor value $(F i c=0.66)$, while the other categories presented Fic values lower than 0.5. These low values reflect the diversity of knowledge displayed by migrants, which can probably be attributed to different cultural influences during their migrations through Brazilian territory. Furthermore, the small number of interviewees may have resulted in low values of Fic. 
Table 3 The 12 categories of use comprising the 41 complaints, their total and partial number of plants cited by the five migrants

\begin{tabular}{|c|c|c|}
\hline Category of use & Complaints (number of plants cited) & $\begin{array}{l}\text { Total number of } \\
\text { plants }\end{array}$ \\
\hline $\begin{array}{l}\text { 1- Gastrointestinal } \\
\text { disturbances }\end{array}$ & $\begin{array}{l}\text { To combat worms (1), ulcer (1), diarrhoea (1), bellyache (2), heartburn (1), intestinal infections (1), liver } \\
\text { pain (3), to improve digestion (1), hemorrhoid (1), as laxative (10) and for stomach purify (2) }\end{array}$ & 24 \\
\hline $\begin{array}{l}\text { 2- Inflammatory } \\
\text { processes }\end{array}$ & $\begin{array}{l}\text { As anti-inflammatory (3) and healing (6), to treat sty/conjunctivitis (2), inflammation in the mouth/throat } \\
\text { (3), rheumatism (2), sinusitis (2) and gingivitis (1) }\end{array}$ & 19 \\
\hline $\begin{array}{l}\text { 3- Respiratory } \\
\text { problems }\end{array}$ & To combat cough (1), bronchitis (15) and as expectorant (2) & 18 \\
\hline $\begin{array}{l}\text { 4- Anxiolytic/ } \\
\text { hypnotics }\end{array}$ & As sedative (11) & 11 \\
\hline $\begin{array}{l}\text { 5-Osteomuscular } \\
\text { problems }\end{array}$ & $\begin{array}{l}\text { To ease back pain (1), muscles pain (6), hip pain (1), prevent osteoporosis (1) and to treat lesions in } \\
\text { bone (1) }\end{array}$ & 10 \\
\hline $\begin{array}{l}\text { 6- Dermatological } \\
\text { problems }\end{array}$ & To combat external allergies (2), wounds in the body (5) and inhibits the growth of skin stains (1) & 8 \\
\hline $\begin{array}{l}\text { 7- Genitourinary } \\
\text { disturbances }\end{array}$ & As diuretic (5), to combat kidney stone (2) and treating urine with blood (1) & 8 \\
\hline 8- Endocrine system & To reduce cholesterol (1) and diabetes (3) & 4 \\
\hline $\begin{array}{l}\text { 9- Cardiovascular } \\
\text { problems }\end{array}$ & Treat heart problems (1) and as blood purifier (2) & 3 \\
\hline $\begin{array}{l}\text { 10- Immunological } \\
\text { problems }\end{array}$ & To combat breast cancer (1) and stomach cancer (1) & 2 \\
\hline 11- Analgesics & Earache (2) & 2 \\
\hline 12- Fever & To combat fever (1) & 1 \\
\hline Total & & $110^{*}$ \\
\hline
\end{tabular}

The parts of the plants most often used in the formulas were leaves $(45.4 \%)$ and other aerial parts $(22.7 \%)$. The most common formula was the infusion (37.8\%), followed by in natura (17.6\%) and syrup (10.1\%). The most cited route of administration was ingestion (51.3\%), followed by inhalation (8.4\%) and topical (3.4\%). Plants with restrictions on use and/or toxic Among the 10 specimens with restrictions on use, 6 were designated as only toxic: "alamanda-amarela"
(Allamanda cathartica), "algodão-do-mato" (Asclepias curassavica L.), "amendoim-bravo/burra-leiteira" (Euphorbia heterophylla L.), "azaléa” (Rhododendron simsii), "comigo-ninguém-pode" (Dieffenbachia sp.) and "impatiens" (Impatiens hawkeri). The interviewees explained that depending on the dose, the latex of "alamanda-amarela" and "amendoim-bravo" can cause discomfort or even blindness. According to Oliveira et al. [44], the leaves of Dieffenbachia picta Schott contain

Table 4 Values of Informant consensus factor (Fic) for each category of use, considering the plants cited by the five Diadema's migrants

\begin{tabular}{|c|c|c|c|c|c|c|}
\hline SN & Category of use & Plant specimen & $\%$ All Species & Use citation & $\%$ All use citation & Fic \\
\hline 1 & Gastrointestinal disturbances & 24 & 30.77 & 44 & 25.29 & 0.46 \\
\hline 2 & Inflammatory processes & 19 & 24.36 & 28 & 16.09 & 0.33 \\
\hline 3 & Respiratory problems & 18 & 23.07 & 31 & 17.82 & 0.43 \\
\hline 4 & Anxiolytic/hypnotics & 11 & 14.10 & 19 & 10.92 & 0.44 \\
\hline 5 & Osteomuscular problems & 10 & 12.82 & 13 & 7.47 & 0.25 \\
\hline 6 & Dermatological problems & 8 & 10.26 & 11 & 6.32 & 0.3 \\
\hline 7 & Genitourinary disturbances & 8 & 10.26 & 13 & 7.47 & 0.41 \\
\hline 8 & Endocrine system & 4 & 5.13 & 5 & 2.87 & 0.25 \\
\hline 9 & Immunological problems & 2 & 2.56 & 4 & 2.30 & 0.66 \\
\hline 10 & Cardiovascular problems & 3 & 3.84 & 3 & 1.72 & 0 \\
\hline 11 & Analgesics & 2 & 2.56 & 2 & 1.15 & 0 \\
\hline 12 & Fever & 1 & 1.28 & 1 & 0.57 & 0 \\
\hline
\end{tabular}


calcium oxalate, which damages the oral mucosa and provokes pain and oedema, while the leaves of Allamanda cathartica contain cardiotonic glycosides and induce intense gastrointestinal disturbances.

Although reported as toxic, the latex of two other plants can be used at low doses to treat breast and stomach cancer: "avelóz" (Euphorbia tirucalli L.) and "jarnaúba" (Synadenium grantii Hook. F.), respectively. The sap of "embaúba" (Cecropia pachystachya Tréc.) was indicated as toxic, but its fruits are used to combat bronchitis. Finally, the seeds of "pucunã" (Fevillea passiflora Vell.) are toxic, being indicated as abortive. In a recent study, Rodrigues [45] also described plants with restrictions of use as reported by three Brazilian cultures: the Krahô Indians use two plants as abortives in a single prescription: "aprytytti" (Acosmium dasycarpum (Vogel) Yakovlev) and "ahkryt" (Anacardium occidentale L.) (Anacardiaceae); their barks are boiled, and the beverage is ingested in at dawn. It is an extremely bitter beverage, rich in tannin and therefore extremely astringent.

\section{Pharmacological data}

As can be seen in Table 2, 57 species (73.1\%) were featured in previous pharmacological studies. For 30 of these species $(52.6 \%)$, the uses cited by the migrants showed some similarity to the investigated effects/ actions, demonstrating concordance between popular knowledge and academic science (marked with an asterisk in Table 2).

Animals used for therapeutic purposes and dynamics of use From the five interviewees, only one (PE2) offered knowledge on the medicinal uses of 12 animals. They belong to four taxonomic classes: Reptilia (6 species), Insects (3), Mammalia (2) and Amphibia (1). However, the interviewee has used only two animals since he arrived in Diadema, the other ten animals fell into disuse because they are not available in this city. The two animals were collected, identified and deposited in the Museum of Zoology-USP: ant (Atta sexdens L.) and cockroach (Periplaneta americana L.). These species belong to the maintenance of use category (highlighted with $\square$ in Table 1). The other ten species therefore belong to the discontinued use category (highlighted with ${ }^{\mathrm{O}}$ in Table 1) which could not be collected. Their identifications were made by PE2 through consulting images from books (as described in Methodology). For three animals (snake, alligator and giant water bug) PE2 could only hesitantly confirm their identity, probably due to the great diversity of these animals in Brazil. Therefore, they are denoted in Table 1 as probably belonging to one of three possible genera.

The animals were used in 14 different medicinal formulas, with the skin most commonly used (33.3\%), followed by whole animal (20.0\%), bone (13.4\%), fat $(6.7 \%)$, rattle (6.7\%), tooth $(6.7 \%)$, anthill $(6.7 \%)$ and turtleshell (6.7\%). Some studies conducted in Brazil show that concomitant data corroborate and sustain these uses [46-50]. The formulas were cited for the treatment of nine complaints, which were grouped into six functional categories, as shown in Table 5. The most commonly cited formula was powder $(66.7 \%)$, followed by in natura $(20 \%)$. The most frequent route of administration was ingestion $(78.6 \%)$.

The most common complaint involved respiratory problems (58.4\%; 7 animals) followed by central nervous system $(8.3 \%)$, inflammatory processes $(8.3 \%)$, dermatological problems $(8.3 \%)$, analgesics $(8.3 \%)$, cardiovascular problems $(8.3 \%)$ as shown in Table 5 . The high humidity of the region (with annual rainfall between 1.000 and $1750 \mathrm{~mm}$ ) [21] is known to lead to bronchitis, cough and asthma. This may explain why so many plants and animals were used to treat respiratory disturbances in Diadema, which has been shown in studies of the Sistema Único de Saúde [51] to be the second largest cause of death in Diadema - 14,4\%.

Many animals have been used for medical purposes since antiquity [52-55]. Despite the existence of several ethnopharmacological studies suggesting the bioactive potential of Brazilian fauna [37,56-61], only marine animals have been investigated by chemical and pharmacological methods [62-64]. No pharmacological data was found in the literature for the five animals identified in the present study: rattlesnake (Crotalus cf. durissus L.), capybara (Hydrochoerus cf. hydrochaeris L.), iguana (Iguana cf. iguana L.), ant (Atta sexdens) and cockroach (Periplaneta americana). The lack of information available on medicinal animal products leads us to conclude that this is a largely unexplored topic in Brazil and that future pharmacological studies should confirm the potential therapeutic value of these species.

\section{Table 5 The 6 categories of use comprising the 9 complaints, their respective number of animals mentioned by the migrant PE2}

\begin{tabular}{ll}
\hline Category of use & Complaints (number of animals) \\
\hline 1-Respiratory problems & bronchitis (7), asthma (4) \\
\hline 2-Central nervous system & epilepsy (1) \\
\hline 3-Inflammatory processes & rheumatism (1) \\
\hline 4-Dermatological problems & wounds in the body (1), skin cancer (1) \\
\hline 5-Analgesics & back pain (1) \\
\hline 6-Cardiovascular problems & treat heart problems (1), hemorrhage (1) \\
\hline Total & $\mathbf{1 8}^{*}$
\end{tabular}

* some animals have been cited for more than one complaint, so their total number above (18) is higher than the number of animals indicated: 12 . 


\section{Conclusion}

The migrant interviewees demonstrated knowledge about the medicinal and toxic properties of plants and animals available in the Atlantic Forest remnants of the municipality of Diadema. Migration contributed to the expansion of knowledge regarding the use of natural resources, especially through the processes of resource replacement and/or incorporation. Moreover, the maintenance of original uses of certain resources demonstrates their value in the migrants' therapeutic practices.

The seven plants [Impatiens hawkeri W. Bull., Artemisia canphorata Vill., Equisetum arvensis L., Senna pendula (Humb. \& Bonpl. ex Willd.) H.S. Irwin \& Barneby, Zea mays L., Fevillea passiflora Vell. and Croton fuscescens Spreng)] and the two animals (Atta sexdens and Periplaneta americana) that showed maintenance of use among migrants during their displacement in Brazilian territory, have not been studied by pharmacologists yet. These species should be highlighted in further investigations because the maintenance of use during human migrations can be indicative of bioactive potential.

This work also demonstrates the impossibility of sharing benefits related to property rights with cultures under certain circumstances, as the dynamic use of natural resources presents particularly varied influences. The interviewed migrants had passed through several Brazilian cities and were exposed to distinct vegetation and cultures. In this migration, they have passed on and incorporated knowledge in an intensive exchange where formulas and uses are mixed and re-invented as a result of contact between cultures.

\section{Acknowledgements}

We thank the interviewees for their hospitality, help, and mainly for providing us with information for the purpose of this study. We are grateful to Julino Assunção Rodrigues Soares Neto, Valéria Basti, Maria Conceição D. A. Fernandes. We also appreciate the help of FAPESP (Fundação de Amparo à Pesquisa do Estado de São Paulo), FIC (Faculdade Integral Cantareira) and AFIP (Associação Fundo de Incentivo à Psicofarmacologia), Herbário Municipal de São Paulo (PMSP), which provided financial support which made this research possible. Finally, we thank Dr. Lúcia Rossi and Prof. Dr. Hussam El Dine Zaher, for conducting the botanical and animal identification, respectively.

\section{Author details}

${ }^{1}$ Department of Biology, Universidade Federal de São Paulo, Rua Arthur Ridel, 275 CEP, 09941-510, Diadema, S.P., Brazil. ${ }^{2}$ Department of Psychobiology, Universidade Federal de São Paulo, Rua Botucatu, 862 - 10 andar - Edifício Biomédicas CEP 04023-062, São Paulo, S.P., Brazil.

\section{Authors' contributions}

Author DG performed the fieldwork. Author MVD identified the animal specimens. Author ER supervised the research works. All authors drafted, wrote, read and approved the final manuscript.

\section{Competing interests}

The authors declare that they have no competing interests.

Received: 24 June 2010 Accepted: 29 October 2010 Published: 29 October 2010
References

1. Pieroni A, Vandebroek I: Traveling cultures and plants: the ethnobiology and ethnopharmacy of human migrations Berghahn Books: New York; 2007.

2. Pieroni A, Quave CL: Traditional pharmacopoeias and medicines among Albanians and Italians in southern Italy: a comparison. J Ethnopharmacol 2005, 101:258-270

3. Waldstein A: Mexican migrant ethnopharmacology: pharmacopoeia, classification of medicines and explanations of efficacy. J Ethnopharmacol 2006, 108:299-310.

4. Waldstein A: "Diaspora and Health? Traditional Medicine and Culture in a Mexican Migrant Community". Int Migr 2008, 46:95-117.

5. Volpato G, Godínez D, Beyra A, Barreto : A Uses of medicinal plants by Haitian immigrants and their descendants in the Province of Camagüey, Cuba. J Ethnobiol Ethnomed 2009, 5:16.

6. Voeks RA: Traditions in transition: African diaspora ethnobotany in lowland South America. In Mobility and Migration in Indigenous Amazonia: Contemporary Ethnoecological Perspectives. Edited by: Alexiades M. London: Berghahn; 2009:275-294.

7. Carney J, Voeks RA: Landscape legades of the African Diaspora in Brazil. Prog Hum Geogr 2003, 27:6.

8. Ceuterick M, Vandebroek I, Torry B, Pieroni A: Cross-cultural adaptation in urban ethnobotany: the Colombian folk pharmacopoeia in London. $J$ Ethnopharmacol 2008, 120:342-359.

9. van Andel, P Westers: Why Surinamese migrants in the Netherlands continue to use medicinal herbs from their home country. J Ethnopharmacol 2010, 127:694-701.

10. Pieroni A, Nebel C, Quave $\mathrm{CL}$, Münz $\mathrm{H}$, Heinrich M: Ethnopharmacology of liakra, traditional weedy vegetables of the Arbëreshë of the Vulture area in southern Italy. J Ethnopharmacol 2002, 81:165-185.

11. Pieroni A, Quave CL, Nebel S, Heinrich M: Ethnopharmacy of ethnic Albanians (Arbëreshë) in northern Basilicata (southern Italy). Fitoterapia 2002, 73:217-241.

12. Pieroni A, Quave CL, Villanelli ML, Mangino P, Sabbatini G, Santini L: Ethnopharmacognostic survey on the natural ingredients used in folk cosmetics, cosmeceuticals and remedies for healing skin diseases in the inland Marches, Central-Eastern Italy. J Ethnopharmacol 2004, 91:331-344.

13. Cano $\mathrm{JH}$, Volpato $\mathrm{G}$ : Herbal mixtures in the traditional medicine of Eastern Cuba. J Ethnopharmacol 2004, 90:293-316.

14. Rodrigues E, Mendes FR, Negri G: Plants indicated by Brazilian Indians to Central Nervous System disturbances: a bibliographical approach. Curr Med Chem 2005, 6:211-244.

15. IBGE. [http://www.ibge.gov.br/home/].

16. Instituto Socioambiental. [http://www.socioambiental.org/]

17. Fundação Cultural Palmares. [http://palmares.gov.br/].

18. Giulietti AM, Harley RM, Queiroz LP, Wanderley MGL, Van den Berg C: Biodiversidade e conservação das plantas no Brasil. Megadiversidade 2005, 1:52-61.

19. Marques AC, Lamas CJE: Taxonomia zoológica no Brasil: estado da arte, expectativas e sugestões de ações futuras. Papéis Avulsos de Zoologia 2005, 46:139-174

20. Simões LL, Lino CF: Sustentável Mata Atlântica: a exploração de seus recursos florestais SENAC: São Paulo; 2004.

21. IBAMA. [http://ibama.gov.br/ecossistemas/mata_atlantica.htm]

22. Prefeitura de Diadema. [http://www.diadema.sp.gov.br/apache2-default/].

23. Atlas de Desenvolvimento Humano/PNUD. [http://www.pnud.org.br/atlas/ ].

24. Bernard RH: Research methods in cultural anthropology Sage publications: London; 1988.

25. Lipp FJ: Methods for ethnopharmacological field work. J Ethnopharmacol 1989, 25:139-150.

26. Freitas MA, Silva TFS: A herpetofauna da Mata Atlântica nordestina USEB: Pelotas; 2005.

27. Freitas MA, Silva TFS: A herpetofauna das caatingas e áreas de altitudes do nordeste Brasileiro USEB: Pelotas; 2007.

28. Lorenzi H, Matos FJ, de A: Plantas medicinais do Brasil: nativas e exóticas cultivadas Instituto Plantarum: São Paulo; 2008.

29. PUBMED. [http://www.ncbi.n/m.nih.gov/pubmed].

30. SCIFINDER. [http://www.cas.org/products/sfacad/index.html].

31. Pio Corrêa M: Dicionário das plantas úteis do Brasil e das exóticas cultivadas Imprensa Nacional: Rio de Janeiro; 1926. 
32. Trotter RT, Logan MH: Informant consensus: a new approach for identifying potentially effective medicinal plants. In Plants in indigenous medicine and diet: biobehavioral approachs. Edited by: Etkin NL. New York: Redgrave Publishing; 1986:91-112.

33. Heinrich M: Ethnobotany and its role in drug development. Phytother Res 2000, 14:479-488.

34. Di Stasi LC, Oliveira GP, Carvalhares MA, Queiroz-Junior M, Tien OS, Kakinami SH, Reis MS: Medicinal plants popularly used in the Brazilian Tropical Atlantic Forest. Fitoterapia 2002, 73:69-91.

35. Almeida C, Albuquerque UP: Uso e conservação de plantas e animais medicinais no estado de Pernambuco (nordeste do Brasil): um estudo de caso. Interciencia 2002, 27:276-285.

36. Begossi A, Leitão-Filho HF, Richerson PJ: Plant uses a Brazilian coastal fishing community (Búzios Island). J Ethnobiol Ethnomed 1993, 13:233-256.

37. Rodrigues E: Ethnopharmacology in the Jaú National Park (JNP), state of Amazonas, Brazil. Phytother Res 2006, 5:378-391.

38. Rodrigues E, Carlini EA: Plants used by a Quilombola group in Brazil with potential central nervous system effects. Phytother Res 2004, 18:748-753.

39. Rodrigues E, Carlini EA: Ritual use of plants with possible action on the central nervous system by the Kraho indians, Brazil. Phytother Res 2005, 19:129-135.

40. Díaz JL: Ethnopharmacology of sacred psychoactive plants used by the Indians of Mexico. Pharmacol Toxicol 1977, 17:647-675.

41. Woodland DW: Contemporary plant systematics Andrews University Press: London; 1997.

42. Schultes RE, Raffaulf RF: In The Healing Forest. Medicinal and Toxic Plants of the Nortwest Amazonia. Volume 2. Dioscorides Press: Oregon; 1990.

43. Pires JM, Mendes FR, Negri G, Duarte-Almeida JM, Carlini EA: Antinociceptive peripheral effect of Achillea millefolium L. and Artemisia vulgaris L.: Both plants known popularly by Brand Names of analgesic drugs. Phytother Res 2009, 23:212-219.

44. Oliveira RB, Godoy SAP, Costa FB: Plantas Tóxicas: Conhecimento e prevenção de acidentes Holos: São Paulo; 2003.

45. Rodrigues E: Plants of restricted use indicated by three cultures in Brazil (caboclo-river dweller, Indian and Quilombola). J Ethnopharmacol 2007, 111:295-302

46. Costa-Neto EM: Animal-based medicines: biological prospection and the sustainable use of zootherapeutic resources. Annals of the Brazilian Academy of Sciences 2005, 77:33-43.

47. Santos-Fita D, Costa-Neto EM: As interações entre os seres humanos e os animais: a contribuição da etnozoologia. Biotemas 2007, 20(4):99-110.

48. Ferreira FS, Brito SV, Ribeiro SC, Saraiva AAF, Almeida WO, Alves RRN: Animal-based folk remedies sold in public markets in Crato and Juazeiro do Norte, Ceará, Brazil. BMC Complement Altern Med 2009, 9:17.

49. Alves RRN: Fauna used in popular medicine in Northeast Brazil. $J$ Ethnobiol Ethnomed 2009, 5:1.

50. Torres DF, de Oliveira ES, Alves RRN, Vasconcellos A: Etnobotânica e etnozoologia em unidades de conservação: uso da biodiversidade na APA de Genipabu, Rio Grande do Norte, Brasil. Interciencia 2009, 34:623-629.

51. Sistema Único de Saúde. [http://tabnet.datasus.gov.br/tabdata/cadernos/ cadernosmap.htm]

52. Gudger EW: Stitching wounds with the mandibles of ants and beetles. J Am Med Assoc 1925, 84:1861-1864.

53. Weiss HB: Entomological medicaments of the past. Journal of the New York Entomological Society 1947, 55:155-168.

54. Conconi JR, Pino JM: The utilization of insects in the empirical medicine of ancient Mexicans. J Ethnobiol Ethnomed 1988, 8:195-202.

55. Antonio TMF, (Eds): Insects as remedies for illnesses in Zaire. The Food Insects Newsletter 1994, 7:4-5.

56. Costa-Neto EM: The use of insects in folk medicine in the state of Bahia, northeastern Brazil, with notes on insects reported elsewhere in Brazilian folk medicine. Hum Ecol 2002, 30:245-263.

57. Alves RRN, Rosa IL: Why study the use of animal products in traditional medicines? J Ethnobiol Ethnomed 2005, 30:1-5.

58. Alves RRN, Delima YCC: Snakes used in ethnomedicine in northeast Brazil environment, development and sustainability. CAB Abstr Lite 2006, 9:455-464

59. Costa-Neto EM: Os moluscos na zooterapia: medicina tradicional e importância clínico-farmacológica. Biotemas 2006, 19:71-78.
60. Hanazaki N, Alves RRN, Begossi A: Hunting and use of terrestrial fauna used by Caiçaras from the Atlantic Forest coast (Brazil). J Ethnobiol Ethnomed 2009, 5:36.

61. Alves RRN, Dias TLP: Usos de invertebrados na medicina popular no Brasil e suas implicações para conservação. Tropical Conservation Science 2010, 2:159-174.

62. Berlink RGS, Hajdu E, Rocha RM, Oliveira JHLL, Hernandez ILC, Seleghim MHR, Granato AC, Almeida EVR, Nunnez CV, Muricy G, Peixinho S, Pessoa C, Moraes MO, Cavalcanti BC, Nascimento GGF, Thiemann OH, Silva M: Challenges and Rewards of Research in Marine Natural Products Chemistry in Brazil. J Nat Prod 2004, 67:510-522.

63. Gray CA, Lira SP, Silva M, Pimenta EF, Thiemann OH, Oliva AG, Hajdu E, Andersen RJ, Berlink RGS: Sulfated Meroterpenoids from the Brazilian Sponge Callyspongia sp. are Inhibitors of the Antileishmaniasis Target Adenosine Phosphoribosyl Transferase. J Org Chem 2006, 71:8685-8690.

64. Kossuga MH, Lira SP, Mchugh S, Torres YR, Lima BA, Veloso K, Ferreira Antonio G, Rocha RM, Berlink RGS: Antibacterial Modified Diketopiperazines from two Ascidians of the Genus Didemnum. J Braz Chem Soc 2009, 20:704-711.

65. Nayak S, Nalabothu P, Sandiford S, Bhogadi V, Adogwa A: Evaluation of wound healing activity of Allamanda cathartica. L. and Laurus nobilis. L. extracts on rats. BMC Complement Altern Med 2006, 12:138-142.

66. Fu Y, Zu Y, Chen L, Efferth T, Liang H, Liu Z, Liu W: Investigation of antibacterial activity of rosemary essential oil against Propionibacterium acnes with atomic force microscopy. Planta Med 2007, 73:1275-1280.

67. Weckesser S, Engel K, Simon-Haarhaus B, Wittmer A, Pelz K, Schempp CM: Screening of plant extracts for antimicrobial activity against bacteria and yeasts with dermatological relevance. Phytomedicine 2007, 14:508-516.

68. Peng CH, Su JD, Chyau CC, Sung TY, Ho SS, Peng CC, Peng RY: Supercritical fluid extracts of rosemary leaves exhibit potent antiinflammation and anti-tumor effects. Biosci Biotechnol Biochem 2007, 71:2223-2232.

69. Nusier MK, Bataineh HN, Daradkah HM: Adverse effects of rosemary (Rosmarinus officinalis L.) on reproductive function in adult male rats. Exp Biol Med 2007, 232:809-813.

70. González-Trujano ME, Peña El, Martínez AL, Moreno J, Guevara-Fefer P, Déciga-Campos M, López-Muñoz FJ: Evaluation of the antinociceptive effect of Rosmarinus officinalis L. using three different experimental models in rodents. J Ethnopharmacol 2007, 22:476-482.

71. Gillij YG, Gleiser RM, Zygadlo JA: Mosquito repellent activity of essential oils of aromatic plants growing in Argentina. Bioresour Technol 2008, 99:2507-2415.

72. Bakirel T, Bakirel U, Keles OU, Ulgen SG, Yardibi H: In vivo assessment of antidiabetic and antioxidant activities of rosemary (Rosmarinus officinalis) in alloxan-diabetic rabbits. J Ethnopharmacol 2008, 28:64-73.

73. Orsi RO, Sforcin JM, Funari SR, Bankova V: Effects of Brazilian and Bulgarian propolis on bactericidal activity of macrophages against Salmonella typhimurium. Int Immunopharmacol 2005, 5:359-368.

74. Fukuda M, Ohkoshi E, Makino M, Fujimoto Y: Studies on the constituents of the leaves of Baccharis dracunculifolia (Asteraceae) and their cytotoxic activity. Chem Pharm Bull 2006, 54:1465-1468.

75. Klopell FC, Lemos M, Sousa JP, Comunello E, Maistro EL, Bastos JK, de Andrade SF: Nerolidol, an antiulcer constituent from the essential oil of Baccharis dracunculifolia DC (Asteraceae). Z Naturforsch C J Biosci 2007, 62:537-542.

76. Da Silva Filho AA, de Sousa JP, Soares S, Furtado NA, Andrade e Silva ML, Cunha WR, Gregório LE, Nanayakkara NP, Bastos JK: Antimicrobial activity of the extract and isolated compounds from Baccharis dracunculifolia D. C. (Asteraceae). Z Naturforsch C J Biosci 2008, 63:40-46.

77. Paulino N, Abreu SR, Uto Y, Koyama D, Nagasawa H, Hori H, Dirsch VM, Vollmar AM, Scremin A, Bretz WA: Anti-inflammatory effects of a bioavailable compound, Artepillin C, in Brazilian propolis. Eur J Pharmacol 2008, 10:296-301.

78. Padilha de Paula J, Gomes-Carneiro MR, Paumgartten FJ: Chemical composition, toxicity and mosquito repellency of Ocimum selloi oil. $J$ Ethnopharmacol 2003, 88:253-260.

79. Kupchan SM, Knox JR, Kelsey JE, Saenzrenauld JA: Calotropin, a cytotoxic principle isolated from Asclepias curassavica L. Science 1964, 25:1685-1686.

80. Radford DJ, Gillies AD, Hinds JA, Duffy P: Naturally occurring cardiac glycosides. Med J Aust 1986, 12:540-544. 
81. De Almeida Barbosa LC, de Alvarenga ES, Demuner AJ, Virtuoso LS, Silva AA: Synthesis of new phytogrowth-inhibitory substituted aryl-pbenzoquinones. Chem Biodivers 2006, 3:553-567.

82. Souza MC, Siani AC, Ramos MF, Menezes-de-Lima OJ, Henriques MG: Evaluation of anti-inflammatory activity of essential oils from two Asteraceae species. Pharmazie 2004, 58:582-586.

83. Schmourlo G, Mendonça-Filho RR, Alviano CS, Costa SS: Screening of antifungal agents using ethanol precipitation and bioautography of medicinal and food plants. J Ethnopharmacol 2005, 15:563-568.

84. De Lima MR, de Souza Luna J, dos Santos AF, de Andrade MC, Sant'Ana AE, Genet JP, Marquez B, Neuville L, Moreau N: Anti-bacterial activity of some Brazilian medicinal plants. J Ethnopharmacol 2006, 21:137-147.

85. Gandhi M, Lal R, Sankaranarayanan A, Sharma PL: Post-coital antifertility action of Ruta graveolens in female rats and hamsters. J Ethnopharmacol 1991, 34:49-59.

86. Oliva A, Meepagala KM, Wedge DE, Harries D, Hale AL, Aliotta G, Duke SO: Natural fungicides from Ruta graveolens L. leaves, including a new quinolone alkaloid. J Agri Food Chem 2003, 12:890-896.

87. Ivanova A, Mikhova B, Najdenski H, Tsvetkova I, Kostova I: Antimicrobial and cytotoxic activity of Ruta graveolens. Fitoterapia 2005, 3:344-347.

88. De Freitas TG, Augusto PM, Montanari T: Effect of Ruta graveolens L. on pregnant mice. Contraception 2005, 71:74-77.

89. Preethi KC, Kuttan G, Kuttan R: Anti-tumour activity of Ruta graveolens extract. Asian Pac J Cancer Prev 2006, 7:439-443.

90. Raghav SK, Gupta B, Agrawal C, Goswami K, Das HR: Anti-inflammatory effect of Ruta graveolens L. in murine macrophage cells. J Ethnopharmacol 2006, 8:234-239.

91. Khori V, Nayebpour M, Semnani S, Golalipour MJ, Marjani A: Prolongation of AV nodal refractoriness by Ruta graveolens in isolated rat hearts. Potential role as an anti-arrhythmic agent. Saudi Med J 2008, 29:357-363.

92. Nogueira JC, Diniz Mde F, Lima EO: In vitro antimicrobial activity of plants in Acute Otitis Externa. Braz J Otorhinolaryngol 2008, 74:118-124.

93. Valadares MC, Carrucha SG, Accorsi W, Queiroz ML: Euphorbia tirucalli L. modulates myelopoiesis and enhances the resistance of tumour-bearing mice. Int Immunopharmacol 2006, 6:294-299.

94. Shlamovitz GZ, Gupta M, Diaz JA: A case of acute keratoconjunctivitis from exposure to latex of Euphorbia tirucalli (Pencil Cactus). J Emerg Med 2007, 36:239-241.

95. Bani S, Kaul A, Khan B, Gupta VK, Satti NK, Suri KA, Qazi GN: Anti-arthritic activity of a biopolymeric fraction from Euphorbia tirucalli. J Ethnopharmacol 2007, 1:92-98.

96. Takahashi H, Hirata S, Minami H, Fukuyama Y: Triterpene and flavanone glycoside from Rhododendron simsii. Phytochemistry 2001, 56:875-879.

97. Frutuoso VS, Gurjão MR, Cordeiro RS, Martins MA: Analgesic and antiulcerogenic effects of a polar extract from leaves of Vernonia condensata. Planta Med 1994, 60:21-25.

98. Valverde AL, Cardoso GL, Pereira NA, Silva AJ, Kuster RM: Analgesic and antiinflammatory activities of vernonioside B2 from Vernonia condensata. Phytother Res 2001, 15:263-264.

99. De Moura RS, Emiliano AF, de Carvalho LC, Souza MA, Guedes DC, Tano T, Resende AC: Antihypertensive and endothelium-dependent vasodilator effects of Alpinia zerumbet, a medicinal plant. J Cardiovasc Pharmacol 2005, 46:288-294.

100. De Araújo PF, Coelho-de-Souza AN, Morais SM, Ferreira SC, Leal-Cardoso JH: Antinociceptive effects of the essential oil of Alpinia zerumbet on mice. Phytomedicine 2005, 12:482-486.

101. Sawangjaroen N, Phongpaichit S, Subhadhirasakul S, Visutthi M, Srisuwan N, Thammapalerd N: The anti-amoebic activity of some medicinal plants used by AIDS patients in southern Thailand. Parasitol Res 2006, 98:588-592.

102. Lin LY, Peng CC, Yeh WT, Wang HE, Yu TH, Peng RY: Alpinia zerumbet potentially elevates high-density lipoprotein cholesterol level in hamsters. J Agric Food Chem 2008, 25:4435-4443.

103. Berson DS: Natural antioxidants. J Drugs Dermatol 2008, 7:7-12.

104. Araújo VT, Diogo DC, da Silva MAP, Riggio LMT, Lapa AJ, Souccar C: Evaluation of the antiurolithiatic activity of the extract of Costus spiralis Roscoe in rats. J Ethnopharmacol 1999, 66:193-198.

105. Palmieri MMB: Efeitos sobre o Sistema Nervoso Central de extratos de plantas popularmente citadas como anticonvulsivantes. MsD thesis Universidade Estadual Paulista, Ribeirão Preto; 2000.
106. Cavalcanti ES, Morais SM, Lima MA, Santana EW: Larvicidal activity of essential oils from Brazilian plants against Aedes aegypti L. Mem Inst Oswaldo Cruz 2004, 99:541-544.

107. Wannissorn B, Jarikasem S, Siriwangchai T, Thubthimthed S: Antibacterial properties of essential oils from Thai medicinal plants. Fitoterapia 2005, 76:233-236.

108. Tchoumbougnang F, Zollo PH, Dagne E, Mekonnen Y: In vivo antimalarial activity of essential oils from Cymbopogon citratus and Ocimum gratissimum on mice infected with Plasmodium berghei. Planta Med 2005, 7:20-23.

109. Moore SJ, Hill N, Ruiz C, Cameron MM: Field evaluation of traditionally used plant-based insect repellents and fumigants against the malaria vector Anopheles darlingi in Riberalta, Bolivian Amazon. J Med Entomol 2007, 44:624-630.

110. Adeneye AA, Agbaje EO: Hypoglycemic and hypolipidemic effects of fresh leaf aqueous extract of Cymbopogon citratus Stapf. in rats. J Ethnopharmacol 2007, 25:440-444.

111. Pintão AM, Pais MS, Coley $H$, Kelland $L R$, Judson IR: In vitro and in vivo antitumor activity of benzyl isothiocyanate: a natural product from Tropaeolum majus. Planta Med 1995, 61:233-236.

112. Soicke $H$, Leng-Peschlow E: Characterisation of flavonoids from Baccharis trimera and their antihepatotoxic properties. Planta Med 1987, 53:37-39.

113. Gené RM, Cartaña C, Adzet T, Marín E, Parella T, Cañigueral S: Antiinflammatory and analgesic activity of Baccharis trimera: identification of its active constituents. Planta Med 1996, 62:232-235.

114. Torres LM, Gamberini MT, Roque NF, Lima-Landman MT, Souccar C, Lapa AJ: Diterpene from Baccharis trimera with a relaxant effect on rat vascular smooth muscle. Phytochemistry 2000, 55:617-619.

115. Januário AH, Santos SL, Marcussi S, Mazzi MV, Pietro RC, Sato DN, Ellena J, Sampaio SV, França SC, Soares AM: Neo-clerodane diterpenoid, a new metalloprotease snake venom inhibitor from Baccharis trimera (Asteraceae): anti-proteolytic and anti-hemorrhagic properties. Chem Biol Interact 2004, 7:243-251.

116. Simões-Pires CA, Queiroz EF, Henriques AT, Hostettmann K: Isolation and on-line identification of antioxidant compounds from three Baccharis species by HPLC-UV-MS/MS with post-column derivatisation. Phytochem Anal 2005, 16:307-314.

117. Oliveira AC, Endringer DC, Amorim LA, das Graças L, Brandão M, Coelho MM: Effect of the extracts and fractions of Baccharis trimera and Syzygium cumini on glycaemia of diabetic and non-diabetic mice. $J$ Ethnopharmacol 2005, 1:465-169.

118. Dickel ML, Rates SM, Ritter MR: Plants popularly used for loosing weight purposes in Porto Alegre, South Brazil. J Ethnopharmacol 2007, 3:60-71.

119. Carvalho LH, Krettli AU: Antimalarial chemotherapy with natural products and chemically defined molecules. Mem Inst Oswaldo Cruz 1991, 2:181-184.

120. Portillo A, Vila R, Freixa B, Adzet T, Cañigueral S: Antifungal activity of Paraguayan plants used in traditional medicine. J Ethnopharmacol 2001, 76:93-98.

121. Molina M, Contreras CM, Tellez-Alcantara P: Mimosa pudica may possess antidepressant actions in the rat. Phytomedicine 1999, 6:319-323.

122. Mahanta M, Mukherjee AK: Neutralisation of lethality, myotoxicity and toxic enzymes of Naja kaouthia venom by Mimosa pudica root extracts. J Ethnopharmacol 2001, 75:55-60.

123. Ngo Bum E, Dawack DL, Schmutz M, Rakotonirina A, Rakotonirina SV, Portet C, Jeker A, Olpe HR, Herrling P: Anticonvulsant activity of Mimosa pudica decoction. Fitoterapia 2004, 75:309-314.

124. Lans C: Ethnomedicines used in Trinidad and Tobago for reproductive problems. J Ethnobiol Ethnomed 2007, 15:3-13.

125. Velázquez E, Tournier HA, Mordujovich de Buschiazzo P, Saavedra G, Schinella GR: Antioxidant activity of Paraguayan plant extracts. Fitoterapia 2003, 74:91-97.

126. Consolini AE, Ragone MI, Migliori GN, Conforti P, Volonté MG: Cardiotonic and sedative effects of Cecropia pachystachya Mart. (ambay) on isolated rat hearts and conscious mice. J Ethnopharmacol 2006, 15:90-96.

127. Schinella G, Aquila S, Dade M, Giner R, Recio Mdel C, Spegazzini E, de Buschiazzo P, Tournier H, Ríos JL: Anti-inflammatory and apoptotic activities of pomolic acid isolated from Cecropia pachystachya. Planta Med 2008, 74:215-220. 
128. Cáceres A, Alvarez AV, Ovando AE, Samayoa BE: Plants used in Guatemala for the treatment of respiratory diseases. 1. Screening of 68 plants against gram-positive bacteria. J Ethnopharmacol 1991, 31:193-208.

129. Pascual ME, Slowing K, Carretero ME, Villar A: Antiulcerogenic activity of Lippia alba (Mill.) N. E. Brown (Verbenaceae). Farmaco 2001, 56:501-504.

130. Zétola M, De Lima TC, Sonaglio D, González-Ortega G, Limberger RP, Petrovick R, Bassani VL: CNS activities of liquid and spray-dried extracts from Lippia alba-Verbenaceae (Brazilian false melissa). J Ethnopharmacol 2002, 82:207-215.

131. Andrighetti-Fröhner CR, Sincero TC, da Silva AC, Savi LA, Gaido CM, Bettega JM, Mancini M, de Almeida MT, Barbosa RA, Farias MR, Barardi CR, Simões CM: Antiviral evaluation of plants from Brazilian Atlantic Tropical Forest. Fitoterapia 2005, 76:374-378.

132. Aridoğan BC, Baydar H, Kaya S, Demirci M, Ozbaşar D, Mumcu E: Antimicrobial activity and chemical composition of some essential oils. Arch Pharm Res 2002, 25:860-864.

133. Choi EM, Hwang JK: Antiinflammatory, analgesic and antioxidant activities of the fruit of Foeniculum vulgare. Fitoterapia 2004, 75:557-565.

134. Lee HS: Acaricidal activity of constituents identified in Foeniculum vulgare fruit oil against Dermatophagoides spp. (Acari: Pyroglyphidae). J Agric Food Chem 2004, 19:2887-2889.

135. Ozcan MM, Chalchat JC, Arslan D, Ates A, Unver A: Comparative essential oil composition and antifungal effect of bitter fennel (Foeniculum vulgare ssp. piperitum) fruit oils obtained during different vegetation. $J$ Med Food 2006, 9:552-561.

136. Tognolini M, Ballabeni V, Bertoni S, Bruni R, Impicciatore M, Barocelli E: Protective effect of Foeniculum vulgare essential oil and anethole in an experimental model of thrombosis. Pharmacol Res 2007, 56:254-260.

137. Pitasawat B, Champakaew D, Choochote W, Jitpakdi A, Chaithong U, Kanjanapothi D, Rattanachanpichai E, Tippawangkosol P, Riyong D, Tuetun B, Chaiyasit D: Aromatic plant-derived essential oil: an alternative larvicide for mosquito control. Fitoterapia 2007, 78:205-210.

138. Gray AM, Flatt PR: Antihyperglycemic actions of Eucalyptus globulus (Eucalyptus) are associated with pancreatic and extra-pancreatic effects in mice. J Nutr 1998, 128:2319-2323.

139. Silva J, Abebeb W, Sousa SM, Duarte VG, Machado MIL, Matos FJA: Analgesic and anti-inflammatory effects of essential oils of Eucalyptus. J Ethnopharmacol 2003, 89:277-283.

140. Takahashi T, Kokubo R, Sakaino M: Antimicrobial activities of eucalyptus leaf extracts and flavonoids from Eucalyptus maculata. Lett Appl Microbiol 2004, 39:60-64.

141. Salari MH, Amine G, Shirazi MH, Hafezi R, Mohammadypour M: Antibacterial effects of Eucalyptus globulus leaf extract on pathogenic bacteria isolated from specimens of patients with respiratory tract disorders. Clin Microbiol Infect 2006, 12:194-196.

142. Zheng YY, Yang J, Chen DH, Sun L: Effects of the stilbene extracts from Cajanus cajan L. on ovariectomy-induced bone loss in rats. Yao Xue Xue Bao 2007, 42:562-565.

143. Braga FG, Bouzada ML, Fabri RL, de O, Matos M, Moreira FO, Scio E, Coimbra ES: Antileishmanial and antifungal activity of plants used in traditional medicine in Brazil. J Ethnopharmacol 2007, 4:396-402.

144. Luo QF, Sun L, Si JY, Chen DH, Du GH: Hypocholesterolemic effect of stilbene extract from Cajanus cajan L. on serum and hepatic lipid in diet-induced hyperlipidemic mice. Yao Xue Xue Bao 2008, 43:145-149.

145. Obaseiki-Ebor EE: Preliminary report on the in vitro antibacterial activity of Bryophyllum pinnatum leaf juice. Afr J Med Med Sci 1985, 14:199-202.

146. Pal S, Nag Chaudhuri AK: Studies on the anti-ulcer activity of a Bryophyllum pinnatum leaf extract in experimental animals. $J$ Ethnopharmacol 1991, 33:97-102.

147. Akinpelu DA: Antimicrobial activity of Bryophyllum pinnatum leaves. Fitoterapia 2000, 71:193-194.

148. Ojewole JA: Antinociceptive, anti-inflammatory and antidiabetic effects of Bryophyllum pinnatum (Crassulaceae) leaf aqueous extract. $J$ Ethnopharmacol 2005, 13:13-19.

149. Yemitan OK, Salahdeen HM: Neurosedative and muscle relaxant activities of aqueous extract of Bryophyllum pinnatum. Fitoterapia 2005, 76:187-193.

150. Penido C, Costa KA, Futuro DO, Paiva SR, Kaplan MA, Figueiredo MR, Henriques MG: Anti-inflammatory and anti-ulcerogenic properties of Stachytarpheta cayennensis (L.C. Rich) Vahl. J Ethnopharmacol 2006, 8:225-233
151. Adebajo AC, Olawode EO, Omobuwajo OR, Adesanya SA, Begrow F, Elkhawad A, Akanmu MA, Edrada R, Proksch P, Klaes M, Verspohl EJ: Hypoglycaemic constituents of Stachytarpheta cayennensis leaf. Planta Med 2007, 7:3241-3250.

152. Anas K, Jayasree PR, Vijayakumar T, Manish Kumar PR: In vitro antibacterial activity of Psidium guajava Linn. leaf extract on clinical isolates of multidrug resistant Staphylococcus aureus. Indian J Exp Biol 2008, 46:41-46.

153. Cheruiyot KR, Olila D, Kateregga J: In-vitro antibacterial activity of selected medicinal plants from Longisa region of Bomet district, Kenya. Afr Health Sci 2009, 1:42-46.

154. Rahim N, Gomes DJ, Watanabe H, Rahman SR, Chomvarin C, Endtz HP, Alam M: Antibacterial activity of Psidium guajava leaf and bark against multidrug-resistant Vibrio cholerae: implication for cholera control. Jpn J Infect Dis 2010, 63:271-274.

155. Roy CK, Das AK: Comparative evaluation of different extracts of leaves of Psidium guajava Linn. for hepatoprotective activity. Pak J Pharm Sci 2010, 23:15-20.

156. Ruppelt BM, Pereira EF, Gonçalves LC, Pereira NA: Pharmacological screening of plants recommended by folk medicine as anti-snake venom-I. Analgesic and anti-inflammatory activities. Mem Inst Oswaldo Cruz 1991, 2:203-205.

157. Soares de Moura R, Costa SS, Jansen JM, Silva CA, Lopes CS, BernardoFilho M, Nascimento da Silva V, Criddle DN, Portela BN, Rubenich LM, Araujo RG, Carvalho LC: Bronchodilator activity of Mikania glomerata Sprengel on human bronchi and guinea-pig trachea. J Pharm Pharmacol 2002, 54:249-256.

158. Maiorano VA, Marcussi S, Daher MA, Oliveira CZ, Couto LB, Gomes OA, França SC, Soares AM, Pereira OS: Antiophidian properties of the aqueous extract of Mikania glomerata. J Ethnopharmacol 2005, 1:364-370.

159. Islam ME, Haque ME, Mosaddik MA: Cytotoxicity and antibacterial activity of Sida rhombifolia (Malvaceae) grown in Bangladesh. Phytother Res 2003, 17:973-975.

160. Dhalwal K, Deshpazde YS, Purohit AP: Evaluation of in vitro antioxidant activity of Sida rhombifolia (L.) ssp. retusa (L.). J Med Food 2007, 10:683-688.

161. Von Szczepanski C, Zgorzelak P, Hoyer GA: Isolation, structural analysis and synthesis of an antimicrobial substance from Petiveria alliacea $\mathrm{L}$. Arzneimittelforschung 1972, 22:1975-1976.

162. Malpezzi EL, Davino SC, Costa LV, Freitas JC, Giesbrecht AM, Roque NF: Antimitotic action of extracts of Petiveria alliacea on sea urchin egg development. Braz J Med Biol Res 1994, 27:749-754.

163. Lopes-Martins RA, Pegoraro DH, Woisky R, Penna SC, Sertié JA: The antiinflammatory and analgesic effects of a crude extract of Petiveria alliacea L. (Phytolaccaceae). Phytomedicine 2002, 9:245-248.

164. Kim S, Kubec R, Musah RA: Antibacterial and antifungal activity of sulfurcontaining compounds from Petiveria alliacea L. J Ethnopharmacol 2006, 8:188-192

165. Okada Y, Tanaka K, Sato E, Okajima H: Antioxidant activity of the new thiosulfinate derivative, S-benzyl phenylmethanethiosulfinate, from Petiveria alliacea L. Org Biomol Chem 2008, 21:1097-1102.

166. Tiwari TN, Varma J, Dubey NK, Chansouria JP, Ali Z: Pharmacological evaluation of some bioactive plant products on albino rats. Hindustan Antibiot Bull 1998, 40:38-41.

167. Runnie I, Salleh MN, Mohamed S, Head RJ, Abeywardena MY: Vasorelaxation induced by common edible tropical plant extracts in isolated rat aorta and mesenteric vascular bed. J Ethnopharmacol 2004, 92:311-316.

168. Ka MH, Choi EH, Chun HS, Lee KG: Antioxidative activity of volatile extracts isolated from Angelica tenuissimae roots, peppermint leaves, pine needles, and sweet flag leaves. J Agric Food Chem 2005, 18:4124-4129.

169. Uawonggul N, Chaveerach A, Thammasirirak S, Arkaravichien T, Chuachan C, Daduang S: Screening of plants acting against Heterometrus laoticus scorpion venom activity on fibroblast cell lysis. J Ethnopharmacol 2006, 16:201-207.

170. Rajesh R, Shivaprasad HV, Gowda CD, Nataraju A, Dhananjaya BL, Vishwanath BS: Comparative study on plant latex proteases and their involvement in hemostasis: a special emphasis on clot inducing and dissolving properties. Planta Med 2007, 73:1061-1067. 
171. Antonio JM, Gracioso JS, Toma W, Lopez LC, Oliveira F, Brito AR: Antiulcerogenic activity of ethanol extract of Solanum variabile (false "jurubeba"). J Ethnopharmacol 2004, 93:83-88.

172. Das NG, Baruah I, Talukdar PK, Das SC: Evaluation of botanicals as repellents against mosquitoes. J Vector Borne Dis 2003, 40:49-53.

173. Chiasson H, Bélanger A, Bostanian N, Vincent C, Poliquin A: Acaricidal properties of Artemisia absinthium and Tanacetum vulgare (Asteraceae) essential oils obtained by three methods of extraction. $J$ Econ Entomol 2001, 94:167-171.

174. Kordali S, Kotan R, Mavi A, Cakir A, Ala A, Yildirim A: Determination of the chemical composition and antioxidant activity of the essential oil of Artemisia dracunculus and of the antifungal and antibacterial activities of Turkish Artemisia absinthium, A. dracunculus, A. santonicum, and A. spicigera essential oils. J Agric Food Chem 2005, 30:9452-9458.

175. Lopes-Lutz D, Alviano DS, Alviano CS, Kolodziejczyk PP: Screening of chemical composition, antimicrobial and antioxidant activities of Artemisia essential oils. Phytochemistry 2008, 69:1732-1738.

176. Rao YK, Fang SH, Tzeng YM: Inhibitory effects of the flavonoids isolated from Waltheria indica on the production of NO, TNF-alpha and IL-12 in activated macrophages. Bio Pharm Bull 2005, 28:912-915.

177. Talbourdet S, Sadick NS, Lazou K, Bonnet-Duquennoy M, Kurfurst R, Neveu $M$, Heusèle C, André P, Schnebert S, Draelos ZD, Perrier E: Modulation of gene expression as a new skin anti-aging strategy. $J$ Drugs Dermatol 2007, 6:25-33.

178. Gopalakrishnan M, Rajasekharasetty MR: Effect of papaya (Carica papaya Linn) on pregnancy and estrous cycle in albino rats of Wistar strain. Indian J Physiol Pharmacol 1978, 22:66-70.

179. Emeruwa AC: Antibacterial substance from Carica papaya fruit extract. $J$ Nat Prod 1982, 45:123-127.

180. Sripanidkulchai B, Wongpanich V, Laupattarakasem P, Suwansaksri J, Jirakulsomchok: Diuretic effects of selected Thai indigenous medicinal plants in rats. J Ethnopharmacol 2001, 75:185-190.

181. Anuar NS, Zahari SS, Taib IA, Rahman MT: Effect of green and ripe Carica papaya epicarp extracts on wound healing and during pregnancy. Food Chem Toxicol 2008, 46:2384-2389.

182. Adeyemi OO, Yemitan OK, Afolabi L: Inhibition of chemically induced inflammation and pain by orally and topically administered leaf extract of Manihot esculenta Crantz in rodents. J Ethnopharmacol 2008, 2:6-11.

183. Nguefack J, Budde BB, Jakobsen M: Five essential oils from aromatic plants of Cameroon: their antibacterial activity and ability to permeabilize the cytoplasmic membrane of Listeria innocua examined by flow cytometry. Lett Appl Microbiol 2004, 39:395-400.

184. Ntonifor NN, Ngufor CA, Kimbi HK, Oben BO: Traditional use of indigenous mosquito-repellents to protect humans against mosquitoes and other insect bites in a rural community of Cameroon. East Afr Med J 2006, 83:553-558.

185. Viyoch J, Pisutthanan N, Faikreua A, Nupangta K, Wangtorpol K, Ngokkuen J: Evaluation of in vitro antimicrobial activity of Thai basil oils and their micro-emulsion formulas against Propionibacterium acnes. Int I Cosmet Sci 2006, 28:125-133.

186. De Almeida I, Alviano DS, Vieira DP, Alves PB, Blank AF, Lopes AH, Alviano CS, Rosa MS: Antigiardial activity of Ocimum basilicum essential oil. Parasitol Res 2007, 101:443-452.

187. Bravo E, Amrani S, Aziz M: Ocimum basilicum ethanolic extract decreases cholesterol synthesis and lipid accumulation in human macrophages. Fitoterapia 2008, 79:515-523.

188. Kusamba C, Byamana K, Mbuyi WM: Antibacterial activity of Mirabilis jalapa seed powder. J Ethnopharmacol 1991, 35:197-199.

189. Shao F, Hu Z, Xiong YM, Huang QZ, Wang CG, Zhu RH, Wang DC: A new antifungal peptide from the seeds of Phytolacca americana: characterization, amino acid sequence and cDNA cloning. Biochim Biophys Acta 1999, 19:262-268.

190. Cáceres A, López B, González S, Berger I, Tada I, Maki J: Plants used in Guatemala for the treatment of protozoal infections. I. Screening of activity to bacteria, fungi and (protozoário) American trypanosomes of 13 native plants. J Ethnopharmaco 1998, 62:195-202.

191. Iwalewa EO, Adewunmi CO, Omisore NO, Adebanji OA, Azike CK, Adigun AO, Adesina OA, Olowoyo OG: Pro- and antioxidant effects and cytoprotective potentials of nine edible vegetables in southwest Nigeria. $J$ Med Food 2005, 8:539-544.
192. Moura AC, Silva EL, Fraga MC, Wanderley AG, Afiatpour P, Maia MB: Antiinflammatory and chronic toxicity study of the leaves of Ageratum conyzoides L. in rats. Phytomedicine 2005, 12:138-142.

193. Singh HP, Batish DR, Kaur S, Kohli RK, Arora K: Phytotoxicity of the volatile monoterpene citronellal against some weeds. Z Naturforsch 2006, 61:334-340.

194. Chah KF, Eze CA, Emuelosi CE, Esimone CO: Antibacterial and wound healing properties of methanolic extracts of some Nigerian medicinal plants. J Ethnopharmacol 2006, 8:164-167.

195. Moreira MD, Picanço MC, Barbosa LC, Guedes RN, Barros EC, Campos MR: Compounds from Ageratum conyzoides: isolation, structural elucidation and insecticidal activity. Pest Manag Sci 2007, 63:615-621.

196. Chiasson H, Bostanian NJ, Vincent C: Insecticidal properties of a Chenopodium-based botanical. J Econ Entomol 2004, 97:1373-1377.

197. Kumar R, Mishra AK, Dubey NK, Tripathi YB: Evaluation of Chenopodium ambrosioides oil as a potential source of antifungal, antiaflatoxigenic and antioxidant activity. Int J Food Microbiol 2007, 10:159-164.

198. Candan F, Unlu M, Tepe B, Daferera D, Polissiou M, Sökmen A, Akpulat HA: Antioxidant and antimicrobial activity of the essential oil and methanol extracts of Achillea millefolium subsp. millefolium Afan. (Asteraceae). $J$ Ethnopharmacol 2003, 87:215-220.

199. Agbor GA, Vinson JA, Oben JE, Ngogang JY: In vitro antioxidant activity of three Piper species. J Herb Pharmacother 2007, 7:49-64.

200. Tabopda TK, Ngoupayo J, Liu J, Mitaine-Offer AC, Tanoli SA, Khan SN, Ali MS, Ngadjui BT, Tsamo E, Lacaille-Dubois MA, Luu B: Bioactive aristolactams from Piper umbellatum. Phytochemistry 2008, 69:1726-1731.

201. Dimo T, Nguelefack TB, Kamtchouing P, Dongo E, Rakotonirina A, Rakotonirina SV: Hypotensive effects of a methanol extract of Bidens pilosa Linn on hypertensive rats. C R Acad Sci Gen 1999, 322:323-329.

202. Chang CL, Kuo HK, Chang SL, Chiang YM, Lee TH, Wu WM, Shyur LF, Yang WC: The distinct effects of a butanol fraction of Bidens pilosa plant extract on the development of Th1-mediated diabetes and Th2mediated airway inflammation in mice. J Biomed Sci 2005, 12:79-89.

203. Sundararajan P, Dey A, Smith A, Doss AG, Rajappan M, Natarajan S: Studies of anticancer and antipyretic activity of Bidens pilosa whole plant. Afr Health Sci 2006, 6:27-30.

204. Rojas JJ, Ochoa VJ, Ocampo AS, Muñoz JF: Screening for antimicrobial activity of ten medicinal plants used in Colombian folkloric medicine: a possible alternative in the treatment of non-nosocomial infections. BMC Complement Altern Med 2006, 6:2.

205. Kviecinski MR, Felipe KB, Schoenfelder T, de Lemos Wiese LP, Rossi MH, Gonçalez E, Felicio JD, Filho DW, Pedrosa RC: Study of the antitumor potential of Bidens pilosa (Asteraceae) used in Brazilian folk medicine. J Ethnopharmacol 2008, 17:69-75.

206. Gbeassor M, Kossou Y, Amegbo K, de Souza C, Koumaglo K, Denke A: Antimalarial effects of eight African medicinal plants. J Ethnopharmacol 1989, 25:115-118.

207. Abreu IC, Marinho AS, Paes AM, Freire SM, Olea RS, Borges MO, Borges AC: Hypotensive and vasorelaxant effects of ethanolic extract from Jatropha gossypiifolia L. in rats. Fitoterapia 2003, 74:650-657.

208. Cetin H, Cinbilgel I, Yanikoglu A, Gokceoglu M: Larvicidal activity of some Labiatae (Lamiaceae) plant extracts from Turkey. Phytother Res 2006, 20:1088-1090.

209. Rim IS, Jee $\mathrm{CH}$ : Acaricidal effects of herb essential oils against Dermatophagoides farinae and D. pteronyssinus (Acari: Pyroglyphidae) and qualitative analysis of a herb Mentha pulegium (pennyroyal). Korean J Parasitol 2006, 44:133-138.

210. Pavela R: Insecticidal properties of several essential oils on the house fly (Musca domestica L.). Phytother Res 2008, 22:274-278.

211. Cechinel Filho V, Santos AR, De Campos RO, Miguel OG, Yunes RA, Ferrari F, Messana I, Calixto JB: Chemical and pharmacological studies of Phyllanthus caroliniensis in mice. J Pharm Pharmacol 1996, 48:1231-1236.

212. Pérez-García F, Marín E, Cañigueral S, Adzet T: Anti-inflammatory action of Pluchea sagittalis: involvement of an antioxidant mechanism. Life Sci 1996, 59:2033-2040.

213. Shi M, Chang L, He G: Stimulating action of Carthamus tinctorius L., Angelica sinensis (Oliv.) Diels and Leonurus sibiricus L. on the uterus. Zhongguo Zhong Yao Za Zhi 1995, 20:173-175.

214. Islam MA, Ahmed F, Das AK, Bachar SC: Analgesic and anti-inflammatory activity of Leonurus sibiricus. Fitoterapia 2005, 76:359-362. 
215. Ahmed F, Islam MA, Rahman MM: Antibacterial activity of Leonurus sibiricus aerial parts. Fitoterapia 2006, 77:316-317.

216. Holetz FB, Pessini GL, Sanches NR, Cortez DA, Nakamura CV, Filho BP: Screening of some plants used in the Brazilian folk medicine for the treatment of infectious diseases. Mem Inst Oswaldo Cruz 2002, 97:1027-1031.

217. Sharma P, Mohan L, Srivastava CN: Phytoextract-induced developmental deformities in malaria vector. Bioresour Technol 2006, 97:1599-1604.

218. Schapoval EE, Vargas MR, Chaves CG, Bridi R, Zuanazzi JA, Henriques AT: Antiinflammatory and antinociceptive activities of extracts and isolated compounds from Stachytarpheta cayennensis. J Ethnopharmacol 1998, 60:53-59.

219. Schuldt EZ, CKless K, Simas ME, Farias MR, Ribeiro-Do-Valle RM: Butanolic fraction from Cuphea carthagenensis Jacq McBride relaxes rat thoracic aorta through endothelium-dependent and endothelium-independent mechanisms. J Cardiovasc Pharmacol 2000, 35:234-239.

220. Biavatti MW, Farias C, Curtius F, Brasil LM, Hort S, Schuster L, Leite SN, Prado SR: Preliminary studies on Campomanesia xanthocarpa (Berg.) and Cuphea carthagenensis (Jacq.) J.F. Macbr. aqueous extract: weight control and biochemical parameters. J Ethnopharmacol 2004, 93:385-389.

221. Nishino H, Hayashi T, Arisawa M, Satomi Y, Iwashima A: Antitumorpromoting activity of scopadulcic acid B, isolated from the medicinal plant Scoparia dulcis L. Oncology 1993, 50:100-103.

222. Ratnasooriya WD, Jayakody JR, Premakumara GA, Ediriweera ER: Antioxidant activity of water extract of Scoparia dulcis. Fitoterapia 2005, 76:220-222.

223. Latha M, Ramkumar KM, Pari L, Damodaran PN, Rajeshkannan V, Suresh T: Phytochemical and antimicrobial study of an antidiabetic plant: Scoparia dulcis L. J Med Food 2006, 9:391-394.

doi:10.1186/1746-4269-6-29

Cite this article as: Garcia et al:: Ethnopharmacological survey among migrants living in the Southeast Atlantic Forest of Diadema, São Paulo, Brazil. Journal of Ethnobiology and Ethnomedicine 2010 6:29.

\section{Submit your next manuscript to BioMed Central and take full advantage of:}

- Convenient online submission

- Thorough peer review

- No space constraints or color figure charges

- Immediate publication on acceptance

- Inclusion in PubMed, CAS, Scopus and Google Scholar

- Research which is freely available for redistribution

Submit your manuscript at www.biomedcentral.com/submit
Biomed Central 